\title{
3D Printing Technologies for Flexible Tactile Sensors toward Wearable Electronics and Electronic Skin
}

\author{
Changyong Liu $1,2\left(\mathbb{D}\right.$, Ninggui Huang ${ }^{1,2}$, Feng Xu ${ }^{1,2}$, Junda Tong ${ }^{1,2}$, Zhangwei Chen ${ }^{1,2}$, \\ Xuchun Gui ${ }^{3}$, Yuelong Fu ${ }^{1,2}$ and Changshi Lao 1,2,* \\ 1 Additive Manufacturing Institute, College of Mechatronics \& Control Engineering, Shenzhen University, \\ Shenzhen 518060, China; liuchangyong@aliyun.com (C.L.); 15875539543@163.com (N.H.); \\ 15258511107@163.com (F.X.); tong_oooo@163.com (J.T.); chen@szu.edu.cn (Z.C.); fuyl@sina.cn (Y.F.) \\ 2 Guangdong Provincial Key Laboratory of Micro/Nano Optomechatronics Engineering, \\ Shenzhen University, Shenzhen 518060, China \\ 3 State Key Laboratory of Optoelectronic Materials and Technologies, School of Electronics and Information \\ Technology, Sun Yat-Sen University, Guangzhou 510275, China; guixch@mail.sysu.edu.cn \\ * Correspondence: cslao@szu.edu.cn; Tel.: +86-755-2690-5532
}

Received: 20 May 2018; Accepted: 5 June 2018; Published: 7 June 2018

\begin{abstract}
D printing has attracted a lot of attention in recent years. Over the past three decades, various 3D printing technologies have been developed including photopolymerization-based, materials extrusion-based, sheet lamination-based, binder jetting-based, power bed fusion-based and direct energy deposition-based processes. 3D printing offers unparalleled flexibility and simplicity in the fabrication of highly complex 3D objects. Tactile sensors that emulate human tactile perceptions are used to translate mechanical signals such as force, pressure, strain, shear, torsion, bend, vibration, etc. into electrical signals and play a crucial role toward the realization of wearable electronics and electronic skin. To date, many types of 3D printing technologies have been applied in the manufacturing of various types of tactile sensors including piezoresistive, capacitive and piezoelectric sensors. This review attempts to summarize the current state-of-the-art 3D printing technologies and their applications in tactile sensors for wearable electronics and electronic skin. The applications are categorized into five aspects: 3D-printed molds for microstructuring substrate, electrodes and sensing element; 3D-printed flexible sensor substrate and sensor body for tactile sensors; 3D-printed sensing element; 3D-printed flexible and stretchable electrodes for tactile sensors; and fully 3D-printed tactile sensors. Latest advances in the fabrication of tactile sensors by 3D printing are reviewed and the advantages and limitations of various 3D printing technologies and printable materials are discussed. Finally, future development of 3D-printed tactile sensors is discussed.
\end{abstract}

Keywords: 3D printing; tactile sensors; wearable electronics; electronic skin

\section{Introduction}

Epidermal electronic system or electronic skin has been introduced as a multifunctional electronic system to detect the physiological signals including temperature, pressure, strain and electrophysiological signals by integrating electrodes, various sensors, power supply and communication components onto a flexible and stretchable substrate [1]. These wearable artificial skin systems have important applications in biomedical engineering and health monitoring [1-13]. Among all the components incorporated in an epidermal electronic system, flexible tactile sensors able to detect force, pressure, strain, shear, torsion, bend and vibration produced by human subtle touch, contact and movement play a very important role $[10,14,15]$. They require high sensitivity with the minimum detectable pressure as low as $\sim 5 \mathrm{~Pa}[16,17]$. In addition to sensitivity, 
flexibility, repeatability/reproducibility and frequency responses are also important for the long-term and real-time monitoring of movement [18]. To date, many researchers have introduced various novel sensing materials with specially designed micro/nanostructures and fabrication schemes to fulfill the requirements $[16,18-44]$. These efforts have greatly advanced the field of flexible and wearable tactile sensors.

In the past few years, 3D printing has attracted a lot of attention due to its manufacturing simplicity, attainable complexity of printed parts, increasing types of printable materials, capability of fabricating multi-material objects, tailored microstructures and declining cost. Due to these advantages, 3D printing has been applied in the fabrication of various functional devices including energy storage devices (lithium-ion batteries [45-54], super-capacitors [55] and fuel cells [56,57]), electronic devices [58-60], biomedical devices [61-64], and various sensors [65]. As for flexible tactile sensors, 3D printing has proven to be an easy, cost-effective and scalable fabrication technique as an alternative to conventional fabrication processes, most of which are complex, high-cost and time-consuming. This review attempts to summarize 3D printing applications in tactile sensors. Latest advances on the printing processes and printable materials are reviewed. Advantages and limitations of various 3D printing processes and future development of 3D-printed tactile sensors are discussed.

\section{A Brief Overview of Tactile Sensors}

\subsection{Functional Requirements of Tactile Sensors}

Tactile sensors are used to emulate human tactile perception such as pressure, shear, bend, torsion, strain and vibration signals with pressure range of $5 \mathrm{~Pa}-100 \mathrm{KPa}$ and vibration frequency up to $\sim 400 \mathrm{~Hz}$ [66]. When tactile sensors are subjected to external loads, mechanical signals are translated into electrical signals that can be detected by an external analyzer. To replicate the functionality of human skin, the requirements of wearable tactile sensors include: (1) flexibility, bendability and stretchability so that the tactile sensors are skin-attachable and wearable; (2) high sensitivity so that minimal pressure, strain, shear and torsion can be detected; (3) wide pressure range; (4) high repeatability and reproducibility over cyclic loading and unloading; (5) good linear input-output characteristics; and (6) fast response to dynamic loads. These requirements have posed great challenges for materials and fabrication technologies. Facile, low cost, reproducible and scalable fabrication technologies have always been a major challenge.

\subsection{Sensing Mechanism of Flexible Tactile Sensors}

Pressure sensing can be achieved through four main sensing mechanisms: piezoresistive, piezoelectric, capacitive and field-effect transistor. Among the four sensing mechanisms, conventional silicon metal-oxide semiconductor field-effect transistor based sensors have a high sensitivity, however they are unsuitable for flexible devices due to its inherent rigidity [22]. To address this issue, organic field-effect transistor (OFET) based on stretchable polymers was proposed [67-71]. Very recently, an intrinsically stretchable polymer OFET array with density of 347 transistors per square centimeter was demonstrated [72]. The transistors exhibited charge-carrier mobility comparable to that of amorphous silicon and varied slightly when subjected to $100 \%$ strain for 1000 cycles. However, to date, it is still very difficult to fabricated OFET-based tactile sensors via 3D printing. Thus, 3D-printed tactile sensors have mainly adopted the three other sensing mechanisms. Therefore, we only focus on these three types of tactile sensor and OFET-based sensors are not described in this review. The basic principles of piezoresistive, capacitive and piezoelectric sensing mechanisms are shown in Figure 1. 


\subsubsection{Piezoresistive Sensing}

As for Piezoresistive sensing, a sensing layer made from piezoresistive materials translates mechanical signals into resistive/conductance change and measured by an external circuit. Conventionally, piezoresistive metal or semiconductor foil patterns are used as the sensing element for commercial strain gauges. Despite the high sensitivity and low cost, these commercial strain gauges are fixed directional sensors and only able to measure very small strains (usually less than 5\%) [18]. Thus, they are not suitable for wearable tactile sensors that are multifunctional, multidirectional and require high sensitivity in a wide pressure range. To meet the requirements of flexible tactile sensors for wearable electronics, novel sensing element based various nanomaterials are required. To date, many types of nanomaterials including nanowires (gold and silver) [23,26,73], carbon nanotubes (CNTs) [17-19,44,74-80], polymer micro/nanostructures [16,81], metal nanoparticles [74,82-84] and graphene $[20-22,25,27,28,30-34,36,38,39,41-44,66,75,82,85-95]$ have been applied to the design of flexible tactile sensors. Remarkable progress has been made in improving the sensitivity, flexibility, stretchability, frequency response and durability of tactile sensors. For example, Amjadi et al. [23] fabricated a strain sensor by embedding silver nanowire (AgNWs) networks between two polydimethylsiloxane (PDMS) layers. The strain sensor showed excellent piezoresistivity with tunable gauge factor (2-14) and high stretchability up to $70 \%$.

\subsubsection{Piezoelectric Sensing}

Piezoelectric effect is a widely used mechanism to fabricate various sensors by converting the change of pressure, acceleration, force, strain, etc. into measurable electrical quantity. Conventionally, piezoelectric ceramics, single crystals and ceramic/polymer composites [96-101] were used as the sensing materials. However, inorganic ceramics are inherently brittle and not suitable for flexible tactile sensors. Hence, polymer-based piezoelectric materials such as poly(vinylidene fluoride) (PVDF) and copolymerized poly(vinylidene fluoride-trifluoroethylene) (PVDF-TrFE) have been used for flexible tactile sensors [102,103]. For example, arrays of highly aligned piezoelectric nanofibers of PVDF and PVDF-TrFE fabricated via electrospinning were applied to fabricate flexible pressure sensors and a pressure sensitivity of over $1.1 \mathrm{~V} \cdot \mathrm{KPa}^{-1}$ was achieved [102]. In addition, many novel hybrid nanocomposites were developed such as $\mathrm{PbTiO}_{3} /$ graphene heterostructure [104] and composite films of alkaline niobate $\mathrm{K}_{0.485} \mathrm{Na}_{0.485} \mathrm{Li}_{0.03} \mathrm{NbO}_{3}$ (KNLN) powders aligned in PDMS matrix [105]. However, available piezoelectric polymers are still very limited and functional materials with both excellent piezoelectric properties and sufficient stretchability require further development.

\subsubsection{Capacitive Sensing}

Capacitive sensing relies on the capacitance change of the dielectric layer sandwiched by conductive electrodes. Pressure or strain-induced deformation that brings the electrodes closer results in the increase of capacitance, and vice versa [106-108]. As an example, Lipomi et al. [107] developed a capacitive pressure and strain sensor composed of a silicone elastomer Ecoflex layer as dielectric and two conductive single-walled carbon nanotubes-coated (SWCNTs) PDMS electrodes. Using these soft elastomers as dielectric and conductive electrodes, capacitive tactile sensors with required stretchability can be obtained.

For a capacitive tactile sensor, the structure of elastomeric dielectric layer is crucial to its performance. Originally, the dielectric layer was made of solid elastomers. However, these sensors suffered from low pressure sensitivity and serious hysteresis due to the viscoelastic properties of elastomers. To address this issue, elastomeric dielectric layers with surface microstructures or 3D porous structures were proposed [109]. The existence of voids in these structures could enable the dielectric layer to elastically and reversibly deform on the application of external pressure or force $[6,70,110-118]$. It was found that the sensitivity can be greatly improved, and hysteresis can be greatly reduced. For example, Mannsfeld et al. [106] fabricated microstructured PDMS films using a 
replica molding process. Capacitive sensors were fabricated using the microstructured PDMS film as the dielectric layer. Greatly improved sensitivity was achieved $\left(0.55 \mathrm{KPa}^{-1} \mathrm{vs} .0 .02 \mathrm{KPa}^{-1}\right.$ for unstructured dielectric layer). In addition, stretchable electrodes are also required for capacitive tactile sensors. Percolation electrodes based on various carbon nanomaterials and metal nanoparticles offer both excellent electrical conductivity and stretchability. By combining various microstructured and porous dielectric elastomeric layer and stretchable percolation electrodes, the performance of capacitive tactile sensors have been greatly enhanced. For example, Kwon et al. [110] fabricated a capacitive pressure sensor composed of 3D microporous Ecoflex dielectric layer and two CNTs-Ecoflex nanocomposite films as percolation electrodes. A pressure sensitivity of $0.601 \mathrm{KPa}^{-1}$ was obtained.

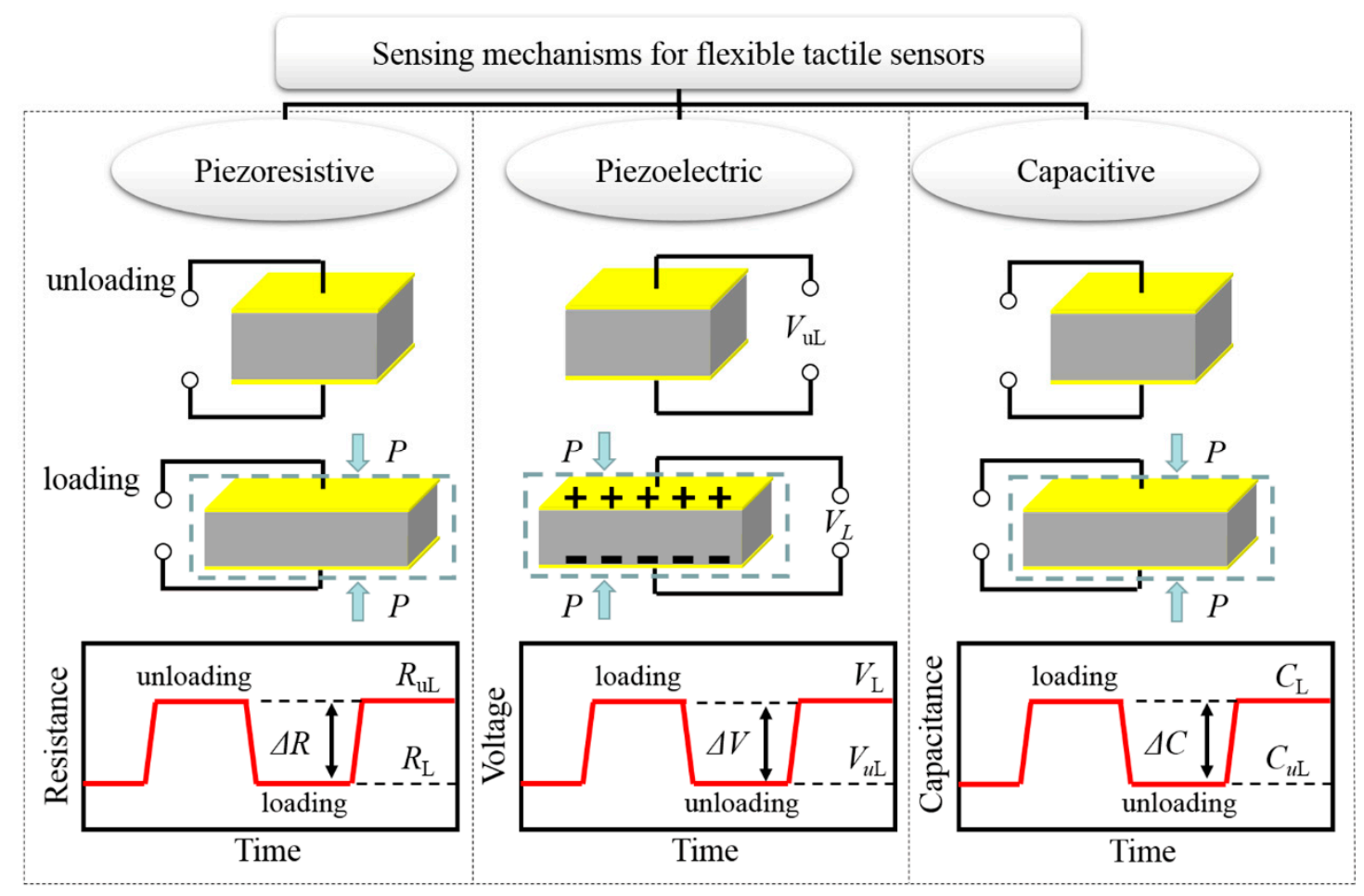

Figure 1. Sensing mechanisms for tactile sensors.

\section{3D printing Applications in Tactile Sensors}

\subsection{A Brief Overview of $3 D$ Printing}

3D printing refers to a group of technologies that fabricate three-dimensional objects directly from computer-aided design (CAD) models in a layer-by-layer fashion [119-121]. With 3D printing, nearly any arbitrarily complex object can be produced in a simple and low-cost manner. To date, many types of 3D printing technologies have been developed. These technologies are usually categorized into photopolymerization-based, sheet lamination-based, materials extrusion-based, power bed fusion-based, direct energy deposition and binder jetting-based technologies. Commonly used 3D printing technologies are shown in Figure 2. There have been many comprehensive reviews on 3D printing and its applications in various fields including metallic parts [120], biomedical engineering [122-126], sensors [65] and microfluidics [127-129]. Therefore, detailed descriptions of these technologies are not given in this review. In recent years, electrohydrodynamic (EHD) 3D printing based on near-field electrospinning (NFES) process has been increasingly applied in the fabrication of micro/nano objects using micro/nanofibers as building blocks [130]. This process has also been used in the fabrication of piezoelectric tactile sensors and is described in this review. 


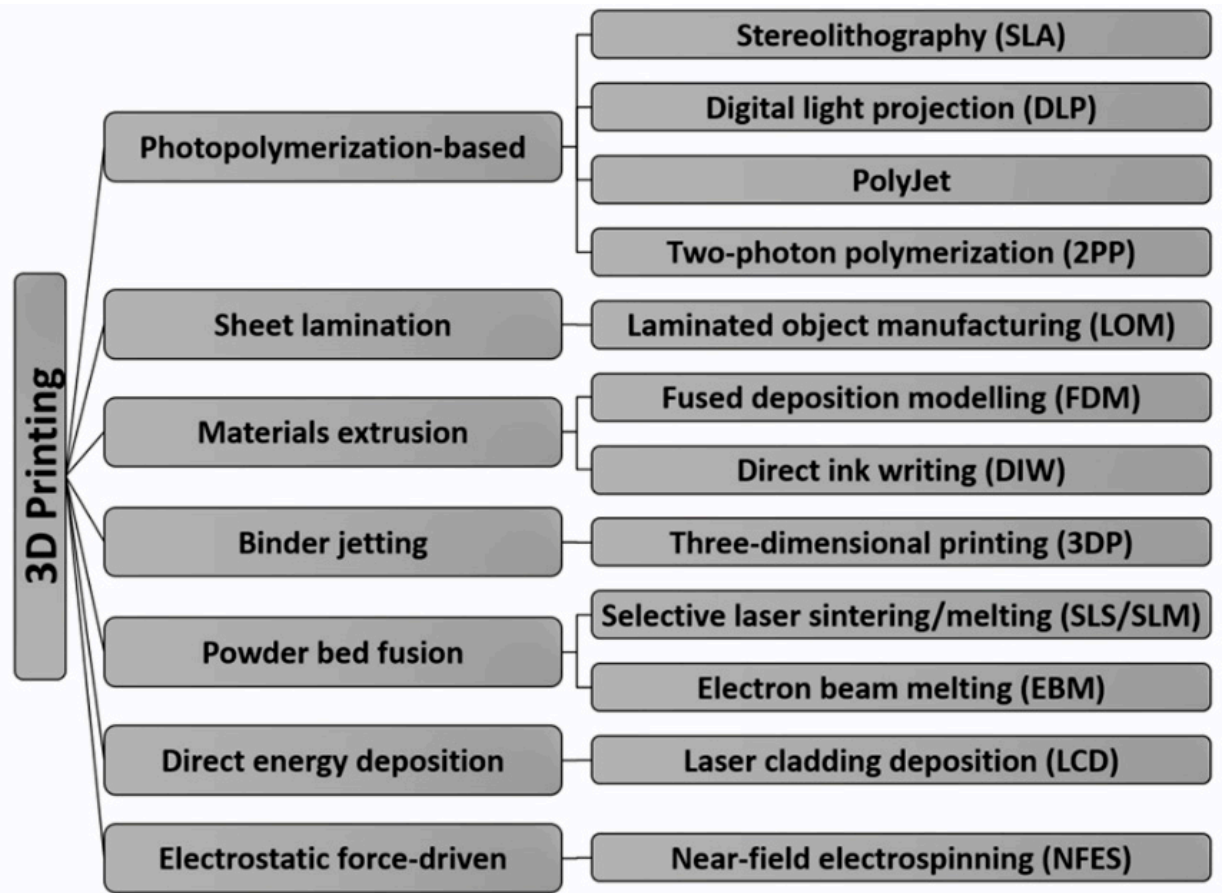

Figure 2. 3D printing technologies.

\subsection{A Brief Overview of 3D Printing Applications in Tactile Sensors}

To date, many researchers have use 3D printing as a powerful tool to address the challenges faced with tactile sensor fabrication. Usually, a tactile sensor is composed of several components including a sensing element, flexible substrate, flexible electrodes, an encapsulation and wiring. Not every tactile sensor has all of the components and the structures of tactile sensors vary from each other. In this review, the 3D printing applications in tactile sensors are categorized into five aspects according to the different types of 3D-printed components.

(1) 3D-printed molds for microstructuring substrate, electrodes and sensing element. For this type of applications, 3D printing is not directly used to fabricate tactile sensor components but used to fabricate molds with various tailored surface structures. These surface structures are replicated by casting elastomeric materials to obtain microstructured substrates, electrodes or sensing elements. With these microstructured components, tactile sensor performance can be improved.

(2) 3D-printed flexible substrate or sensor body to accommodate sensing element. For this type of applications, 3D printing is used to directly fabricate flexible substrate or sensor body using elastomeric materials. Then, the printed substrate and sensor body is used to accommodate sensing element to form a tactile sensor. The major functional requirement for this type of applications is its flexibility and stretchability. To date, many soft silicone rubber-like 3D printable materials have been commercialized and applied to fabricate flexible substrate and sensor body. However, these commercial printable materials still have limited stretchability.

(3) 3D-printed sensing elements for tactile sensors. As for this type of applications, 3D printing is used to fabricate sensing elements with tailored geometries, shapes and microstructures to improve the performance of tactile sensors. Toward this goal, 3D printable sensing materials are required. Usually, these types of materials are not commercially available. Thus, many research groups have developed various types of printable sensing materials such as mixtures of silicone elastomer and conductive fillers as printable piezoresistive inks, printable silicone elastomers as dielectric layer for capacitive tactile sensor and printable piezoelectric polymers for piezoelectric tactile sensors.

(4) 3D-printed flexible electrodes for tactile sensors. In some cases, stretchable electrodes are essential to tactile sensors. For example, a capacitive tactile sensor is usually composed of a pair of 
conductive electrodes and a dielectric layer. Electrodes that are simultaneously electrically conductive and stretchable are highly desired. They should maintain low resistance change upon repeated stretching. 3D printing can be used to fabricate highly stretchable electrodes with a tuned structure such as serpentine design and excellent resistance stability upon stretching can be achieved.

(5) Fully 3D-printed tactile sensors. As for this type of applications, 3D printing is not used to print a single component, but used to print all the components incorporated in a tactile sensor by a specifically designed printing process. Some research groups have proposed such processes to fabricate a whole tactile sensor in one printing procedure.

Table 1 lists representative studies on 3D printing applications in tactile sensors. The 3D printing technology, sensing mechanism, measurable loads, printable materials, materials shape and fabricated components are given. Each study was categorized into these five types of applications according to the fabricated components via 3D printing. To date, the major 3D printing technologies that have been applied to the fabrication of tactile sensors include photopolymerization-based processes, fused deposition modeling (FDM), direct ink writing (DIW) and EHD 3D printing. The following sections describe the four types of 3D printing technologies and their applications in tactile sensors. Other 3D printing processes such as binder jetting, selective laser sintering/melting and direct energy deposition-based processes are seldomly used in tactile sensors and are not described in this review.

\subsection{Photopolymerization-Based 3D Printing for Tactile Sensors}

Photopolymerization-based 3D printing include four types of technologies: stereolithography (SLA), digital light projection (DLP), PolyJet and two-photon polymerization (2PP). SLA typically uses a scanning ultraviolet (UV) laser to cure photocurable resins to form 3D objects layer upon layer (Figure 3a). DLP uses a digital light processing unit (projector) that could enable the exposure and photocuring of a complete layer of resins to form 3D objects (Figure 3b). PolyJet was developed by Stratasys Objet Geometries Co, Ltd. (Eden Prairie, MN, USA) and it uses an inkjet printing-like jetting head with hundreds of micronozzles to eject photocurable resins and supporting materials. Each printed layer was cured by a UV light resource (Figure 3c). After printing, the supporting materials were removed to obtain the printed objects. As for $2 \mathrm{PP}$, the photocuring process of materials was driven not by a single photon, but rather by two photons or multiple photons in combination. The process is typically used to fabricate complex 3D objects with micro/nano-sized features and seldomly used in 3D-printed tactile sensors.
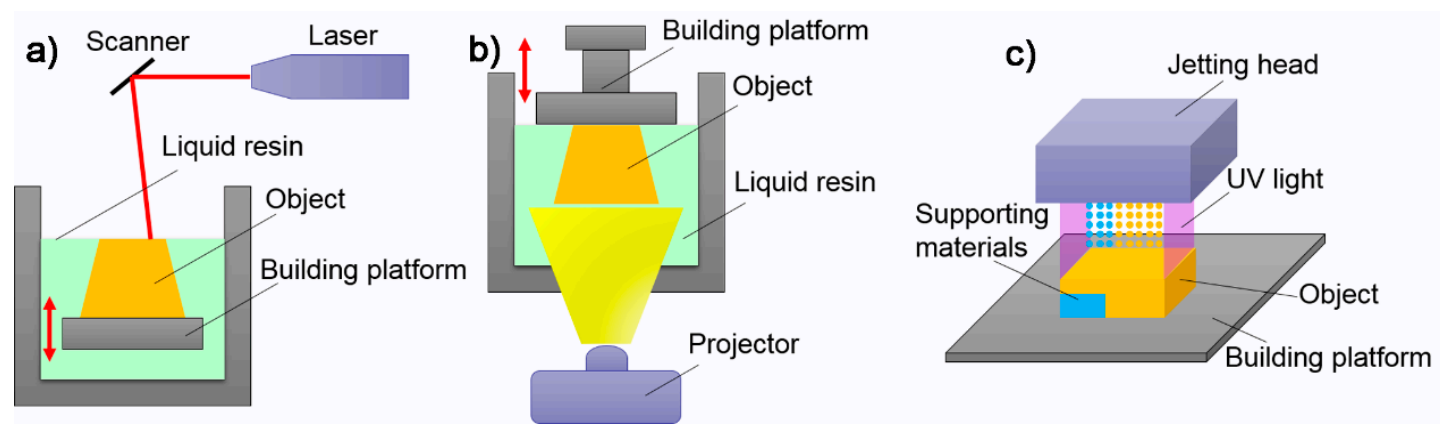

Figure 3. Photopolymerization-based 3D printing: (a) SLA; (b) DLP; and (c) PolyJet.

As for photopolymerization-based processes, the main machine and materials suppliers include 3D System, Formlabs, Stratasys Objet Geometries and Carbon 3D. Initially, these processes were only able to fabricate rigid and brittle parts and they are inappropriate for flexible devices. In recent years, several types of commercially available UV curable rubber-like or elastomeric materials were developed such as Carbon EPU40, Stratasys TangoPlus, Formlabs flexible, 3D System visiJet composite materials. These materials can be used to fabricate flexible and soft-touch prototypes. Some researchers attempted to use these commercially available 3D printing machines and flexible materials to fabricate tactile 
sensors. The fabrication process is shown in Figure 4. An elastomeric sensor body with supporting materials can be fabricated via SLA-, DLP- or PolyJet-based processes. Then the supporting materials were removed and the obtained channels were later filled with piezoresistive materials that functioned as the sensing element. After wiring, tactile sensors can be obtained. Alternatively, the piezoresistive ink can be patterned into the open microchannels using a direct ink writing process.

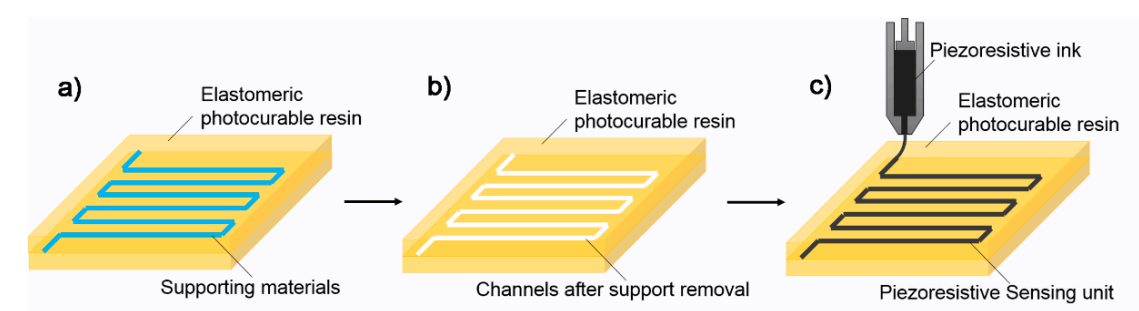

Figure 4. Indirect fabrication process for tactile sensors by photopolymerization-based 3D printing: (a) elastomeric sensor body with supporting materials; (b) elastomeric sensor body after support removal; and (c) piezoresistive ink injection or direct ink writing.

For example, Vatani et al. [131] used PolyJet-based process to fabricate 3D-printed tactile sensors. They designed a tactile sensor consisting of two sensing layers with straight piezoresistive line elements embedded in an elastomeric sensor body. The flexible sensor body was fabricated from a commercially available rubber-like photocurable resin TangoPlus using a PolyJet-based 3D printing machine (Connex 500, Stratasys Objet Co., Ltd., Rehovot, Isreal). Piezoresistive sensing materials were prepared by dispersing multi-walled carbon nanotubes (MWCNTs) into TangoPlus. Then, the obtained piezoresistive TangoPlus/MWCNTs composites were injected or directly printed into/onto the 3D-printed sensor body. The fabricated sensor body and tactile sensors are shown in Figure 5. Similarly, Agarwala et al. [132] used a commercial photopolymerization-based 3D printer (ProJet 5500X, 3D Systems Co., Ltd., Rock Hill, CA, USA) to fabricate a photopolymer matrix with embedded microchannels (500 $\mu \mathrm{m}$ diameter). In the printing process, the microchannels were initially filled with wax as the supporting materials and then washed away after printing. Then, the microchannels were injected with silver conductive ink to act as the piezoresistive sensing unit. The maximum tensile stress and strain were measured as $0.83 \mathrm{MPa}$ and $18.2 \%$, respectively. The gauge factor of the printed sensor was calculated to be 50 at compression strain of $1 \%$.

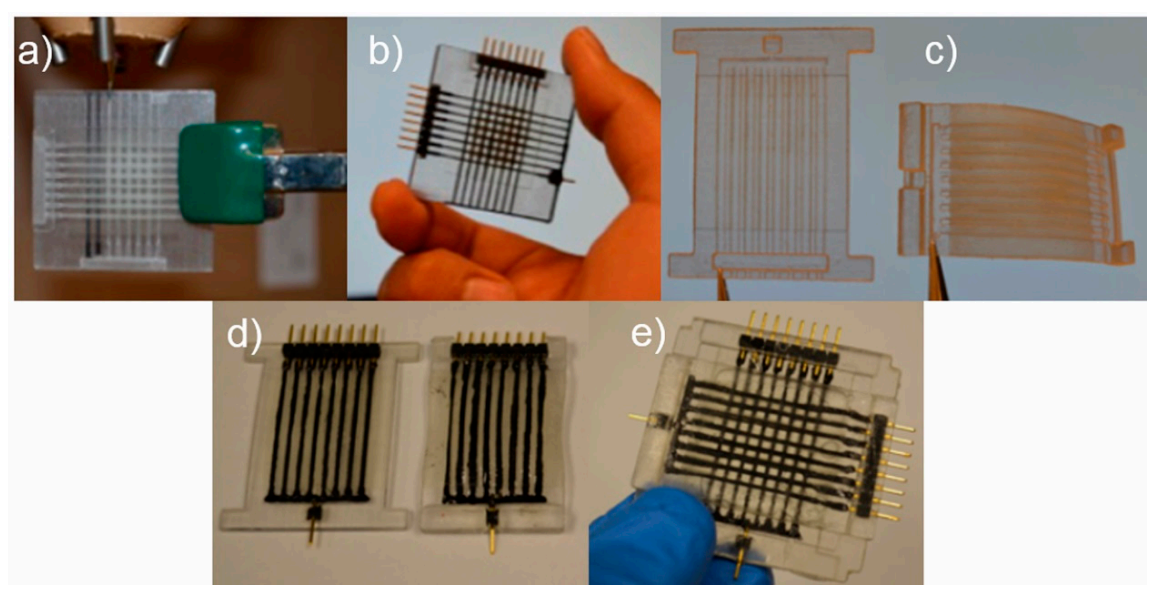

Figure 5. 3D-printed sensor body using photopolymerization-based 3D printing: (a) elastomeric sensor body with internal channels; (b) tactile sensor with injection of piezoresistive ink; (c) top and bottom elastomeric sensor body; (d) direct ink writing of piezoresistive inks on sensor body; and (e) tactile sensor assembly [131]. Reproduced with permission. Copyright Spring Nature, 2015. 
The above studies demonstrated the applications of commercial UV curable elastomers in the fabrication of flexible tactile sensors. However, these elastomers still have great limitations such as insufficient stretchability and inappropriate mechanical properties. To address these issues, Patel et al. [133] developed a family of highly stretchable and UV curable elastomer systems that are suitable for photopolymerization-based 3D printing process including DLP, SLA and PolyJet. The elastomer was prepared by mixing a monofunctional monomer consisting of epoxy aliphatic acrylate (EAA), and a difunctional cross-linker consisting of aliphatic urethane diacrylate (AUD) diluted with $33 \mathrm{wt} \%$ of isobornyl acrylate (EAA-AUD mixture). The developed stretchable and UV curable elastomer was verified by DLP. The printed elastomer can be stretched by $1100 \%$ which was more than five times the elongation at break of commercial UV curable elastomers. In addition, the mechanical properties can be tuned for different applications by varying the EAA-AUD mixing ratio. The authors demonstrated the applications of the elastomer in DLP-printed Bucky ball electronic switches and soft actuators (Figure 6). In comparison with commercially available soft photopolymers, elastomeric sensor body with greatly improved stretchability can be directly fabricated using this new type of materials.

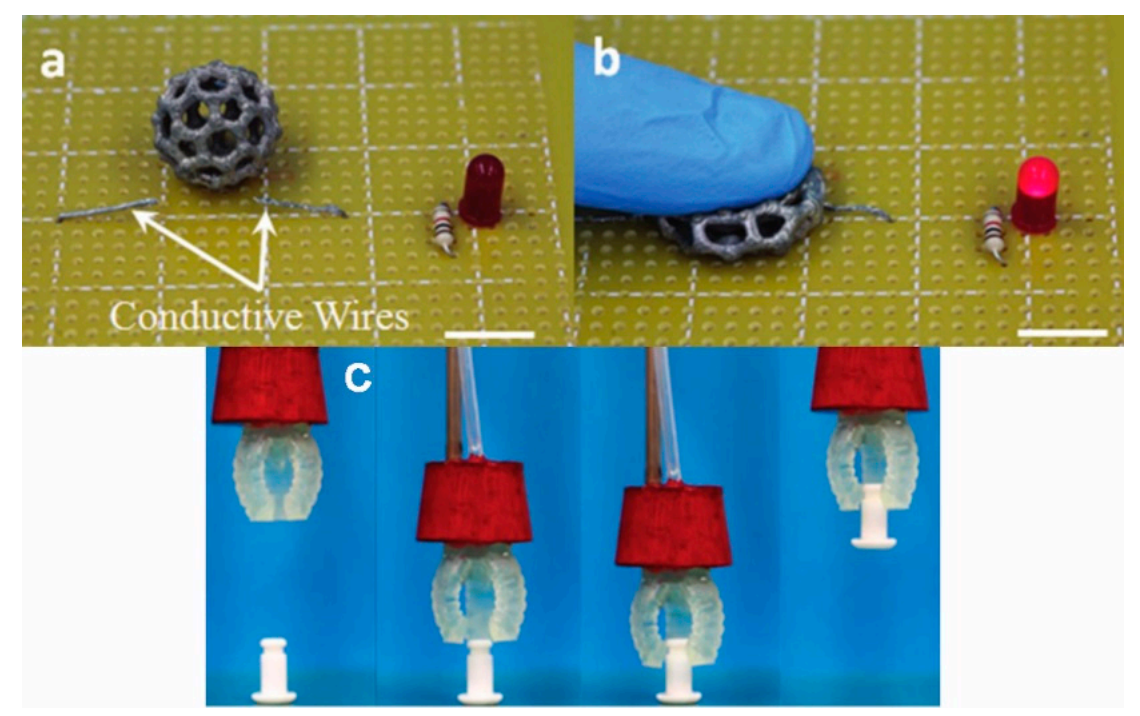

Figure 6. Highly stretchable elastomers fabricated by DLP: (a) DLP-printed Bucky ball coated with silver nanoparticles; (b) a LED turned on after pressing the Bucky ball; and (c) DLP-printed soft robotics [133]. Reproduced with permission. Copyright John Wiley and Sons, 2017.

\subsection{Materials Extrusion-Based 3D Printing for Tactile Sensors}

Fused deposition modeling (FDM) and direct ink writing (DIW) are the main two types of materials extrusion-based 3D printing processes (Figure 7). FDM typically uses a heated nozzle to melt polymer filaments to form 3D objects. While DIW uses a pneumatic nozzle or syringe nozzle to extrude printable inks with appropriate rheological properties to form 3D objects. Both processes have been used to fabricate tactile sensors. As for the FDM process, the printable materials are usually processed into filaments. This is a major limitation for its applications in the fabrication of tactile sensors. As for the DIW process, a wide range of printable inks can be tuned with appropriate rheological properties and offers a unique advantage for tactile sensor fabrication. 

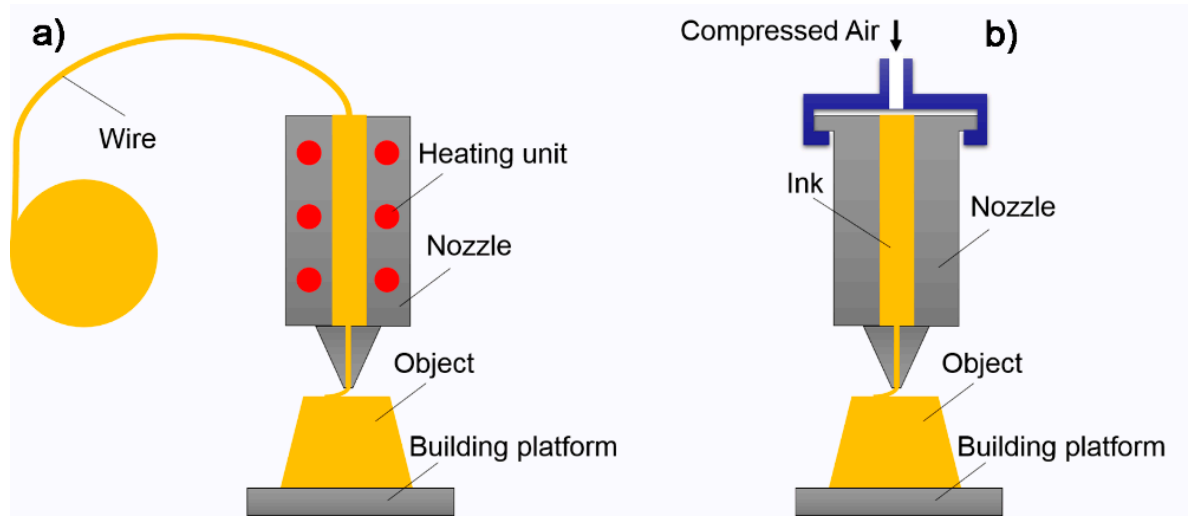

Figure 7. Materials extrusion-based 3D printing technologies: (a) FDM process; and (b) DIW process.

\subsubsection{FDM Process for Tactile Sensors}

The first FDM-printed tactile sensor was demonstrated by Leigh et al. [134] They fabricated flexible piezoresistive and capacitive sensors from polycaprolactone/conductive carbon black (PCL/CB) composite materials using a desktop FDM 3D printer. PCL/CB composites was processed into filaments with a diameter of $1.5 \mathrm{~mm}$ and extruded through a heated nozzle to obtain printed objects. The composite filaments showed piezoresistive properties due to the addition of conductive $\mathrm{CB}$ particles in the PCL matrix. A U-shape piezoresistive sensor was fabricated on a perspex substrate. With the flexing of the substrate, electrical resistance change of the printed sensor was observed. In addition, the authors fabricated a capacitive pressure sensor-based human interface device (HID) using FDM (Figure 8a,b). The HID device can be used to capture the touch sequence by detecting the pressure. These results showed that PCL/CB composites can be processed into filament-type materials for extrusion and tactile sensors may be fabricated via FDM process.

a)

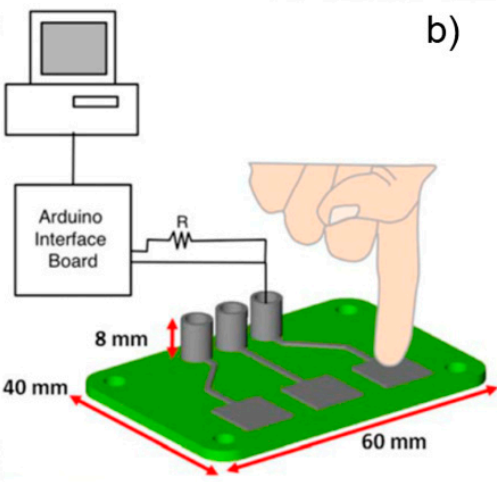

b)

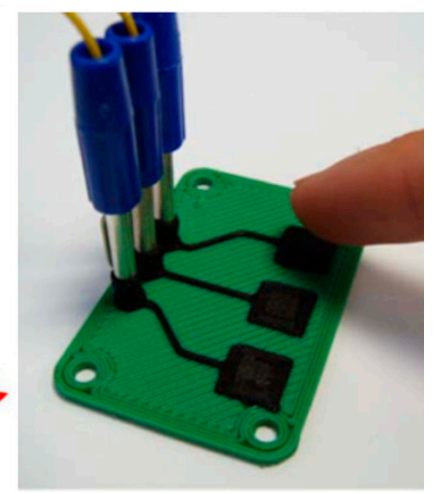

Figure 8. FDM-printed capacitive sensor-based HID using PCL/CB conductive composite: (a) schematic of the device; and (b) 3D-printed capacitive sensors [134].

Saari et al. [135] used FDM process to fabricate capacitive force sensors consisting of 3D-printed transparent ABS-based upper and lower plates, two conductive electrodes and a dielectric layer. The dielectric layer was a $1.27 \mathrm{~mm}$ thick thermoplastic elastomer (TPE, Kraton G1643M, Kraton Performance Polymers Inc., Huston, TX, USA) capacitor core printed by FDM. The conductive electrodes were fabricated using a fiber encapsulation additive manufacturing (FEAM) process. In this process, bare copper wire with the diameter of $127 \mu \mathrm{m}$ was encapsulated by TPE during the extrusion process. The sensitivity of the 3D-printed sensor was measured as $0.215 \pm 0.002 \mathrm{pF} / \mathrm{kN}$. The sensor showed good synchronization between the pressure loads and measured capacitance data. However, $8.3 \mathrm{~s}$ delay was observed due to materials hysteresis. 
Christ et al. [136] prepared thermoplastic polyurethane/multiwalled carbon nanotubes (TPU/MWCNTs) nanocomposite fibers that can be printed by FDM. A piezoresistive strain sensor with dimensions of $1.6 \mathrm{~mm} \times 1.6 \mathrm{~mm} \times 100 \mathrm{~mm}$ was fabricated. The failing strain was $\sim 60 \%$ for the nanocomposites with MWCNTs content of $5 \%$ and a high gauge factor of 176 was obtained. These results demonstrated that FDM-printed TPU/MWCNTs strain sensor exhibited both excellent piezoresistive properties and good stretchability. Kim et al. [137] used a FDM 3D printer with a dual nozzle system that allow the printing of two different materials to fabricate 3D multiaxial force sensor (Figure 9). 3D cubic cross shaped sensors consisting of a structural support and a sensing part were fabricated. The structural part was printed with TPU filament and the sensing part was printed with TPU/CNT nanocomposite filament. The fabricated sensor was able to measure forces along three axes $(x, y$ and $z)$.

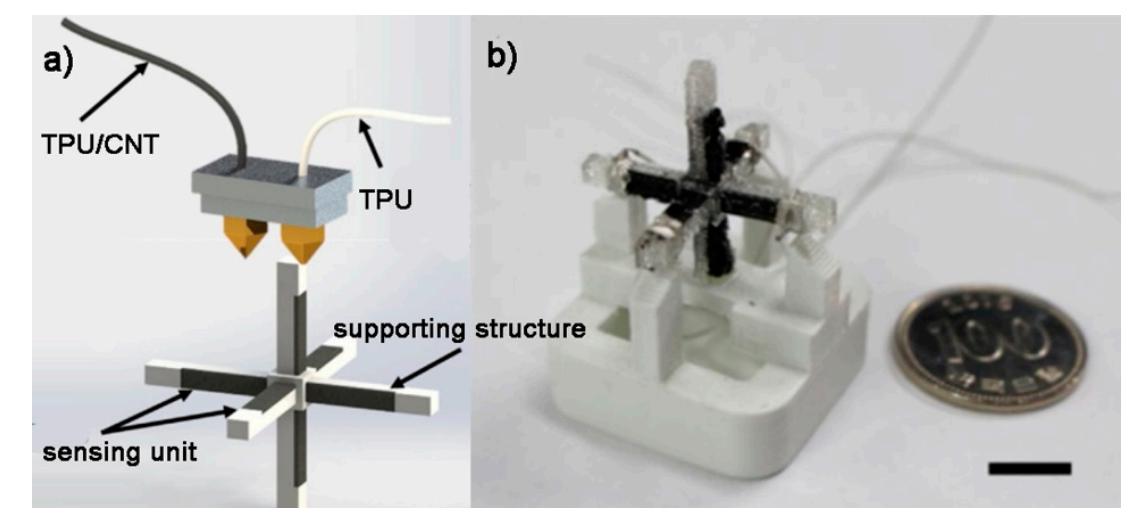

Figure 9. FDM 3D printing for multiaxial force sensors: (a) FDM process with dual nozzles to print TPU and TPU/CNT respectively; and (b) 3D-printed multiaxial force sensor [137]. Reproduced with permission. Copyright Elsevier, 2017.

In addition to directly printing functional components for tactile sensors, FDM can also be used to fabricate molds for microstructuring sensing elements in an indirect approach. For example, Zhuo et al. [138] used a commercial FDM machine to print an ABS mold with tailored surface structures. Then, PDMS was cast onto the mold and microstructured PDMS film with periodical micro-grooves on the surface was obtained and used as the dielectric layer. A capacitive pressure sensor was fabricated by sandwiching the PDMS film by two ITO-coated PET films as electrodes. It was found that the assembled capacitive sensor achieved higher pressure sensitivity than using the micro-fabricated silicon wafer molds.

\subsubsection{Electric Poling-Assisted FDM Process for Tactile Sensors}

FDM can also be used to fabricate piezoelectric tactile sensors using electric poling-assisted FDM process (EPAM) (Figure 10). Lee et al. [139] proposed this new process by combining filament extrusion and applying high electric field between the nozzle tip and substrate. Using this process, piezoelectric polymers such as PVDF filaments can be directly printed into piezoelectric tactile sensors. When a high electrical field was applied to the molten polymer fiber between the nozzle tip and substrate, the PVDF polymer experienced a combinational process of drawing at high temperature, electric poling under high electrical field and annealing at high pressure that contributed to the formation of $\beta$ phase crystalline structure during the printing process. The $\beta$ phase is primarily responsible for the piezoelectric properties of PDVF polymer. The authors found that stronger electric field produced greater piezoelectricity in the printed structures. The measured output current was $\pm 1.5 \mathrm{nA}$ for a single printed layer.

However, the initial EPAM process was only capable of printing pure PVDF filaments and also limited to fabricate only a single layer. In addition, the applied electrical field intensity should not 
exceed $2 \mathrm{MV} \cdot \mathrm{m}^{-1}$, otherwise it may cause the printer to lose communication with the computer. To overcome these limitations, Kim et al. [140] developed an enhanced EPAM process which could enable the application of higher electrical field up to $40 \mathrm{MV} \cdot \mathrm{m}^{-1}$ and the fabrication of multiple layers. With improved electrical field intensity, the authors fabricated piezoelectric pressure sensors using composites of PVDF matrix and $\mathrm{BaTiO}_{3}$ (BTO) fillers to achieve better polarization and enhanced piezoelectric properties. The BTO/PVDF composites with different BTO loading content ranging from $3 \%$ to $15 \%$ were prepared. The authors measured the piezoelectric coefficients of nonpoled PVDF, poled PVDF and BTO/PVDF composite with different BTO content. It was found that the highest amount of $55.91 \% \beta$ phase PVDF was obtained when the BTO content was $15 \%$. The piezoelectric coefficient of $15 \mathrm{wt} \% \mathrm{BTO} / \mathrm{PVDF}$ composite was $0.101 \mathrm{pC} / \mathrm{N}$ and $~ 1300 \%$ larger than nonpoled PVDF. Results showed that BTO filling and in-situ poling could effectively improve the piezoelectric properties.

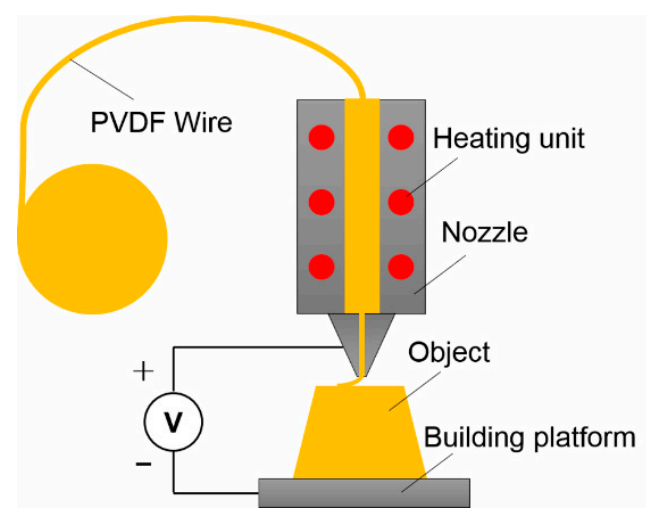

Figure 10. Electric poling-assisted FDM process for tactile sensors.

\subsubsection{Direct Ink Writing for Tactile Sensors}

As for the DIW process, the preparation of a printable ink is a crucial step. A printable ink is usually endowed with appropriate rheological properties and extruded through a nozzle tip using pressurized air or a syringe. The shape and mechanical integrity of printed structures must be maintained during the printing process. Toward this goal, many researchers have developed various printable inks. Most of the printable inks for tactile sensors are mixtures of conductive fillers and stretchable elastomers. Conductive fillers include carbon-based nanomaterials (carbon black, carbon nanotubes, graphene, etc.) and metal-based nanomaterials (metal nanoparticles and nanowires). Silicone elastomers such as Ecoflex and PDMS are the most commonly used matrix materials. In addition, the concept of "ionic skin" using stimuli-responsive hydrogels to fabricate flexible tactile sensors with unique biocompatibility was proposed. DIW can also be used to fabricate ionic tactile sensors using biocompatible hydrogels as printable inks.

In conventional DIW process, printable inks are directly deposited onto the substrate. Some research groups proposed different forms of DIW processes. For example, printable inks can be deposited into a reservoir filled with a second medium. For tactile sensors, piezoresistive inks can be directly printed into a silicone elastomer reservoir and encapsulated within the elastomeric matrix immediately. This process was named embedded DIW process. Another different form of DIW process is the multicore-shell DIW process. In this process, multiple layers of printable inks including a core, middle layers and an encapsulation layer can be coextruded through a nozzle to form a multi-layered tactile sensor in one printing procedure. All the processes have been applied to the fabrication of tactile sensors. 


\section{(1) Conventional DIW Process}

Guo et al. [141] proposed a piezoresistive tactile sensor design composed of the base layer, bottom electrode, isolating layer, supporting layer, sensing layer and top electrode. All of the components in this tactile sensor are fabricated via DIW. The base layer and isolating layer were fabricated using modified silicone ink: DragonSkin 10 with the addition of a thickening agent and curing retarder. The top and bottom electrodes were fabricated from mixtures of silicone elastomer and silver nanoparticles (AgNP) as printable inks. The electrical conductivity of the silicone/AgNP ink increased with the loading of AgNP and the percolation threshold was found to be $67.45 \%$. The top and bottom electrode was printed with $75 \mathrm{wt} \% \mathrm{AgNP} /$ silicone inks given the good balance of high conductivity and stretchability. The sensing layer was printed with $68 \mathrm{wt} \% \mathrm{AgNP} /$ silicone ink since it has the best piezoresistive properties. The supporting layer was printed using a $40 \mathrm{wt} \%$ pluronic ink and later washed away by water. The fabrication process is shown in Figure 11. As for the sensing layer, the current change $\left(\Delta I / I_{0}\right)$ was measured to be $2500 \%, 8000 \%$ and $17,000 \%$ under 60 , 120 and $250 \mathrm{KPa}$ cyclic pressure loads, respectively. For the top and bottom electrodes, the current change was $280 \%$ when $250 \mathrm{KPa}$ pressure load was applied. The printed sensors showed good linear current-voltage characteristics. The gauge factor of the entire printed device was found to be $\sim 180$. As for the frequency response, hysteresis was negligible when the frequency was below $0.25 \mathrm{~Hz}$, while a relative hysteresis of $82 \%$ was observed when the frequency was $1 \mathrm{~Hz}$ due to the viscoelastic properties of silicone. The authors also demonstrated the applications of printed sensors in measuring heartbeat, finger pressure on keyboard and pressure distribution based on sensor arrays.

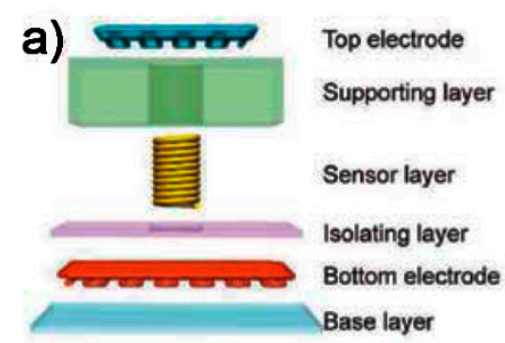

c)
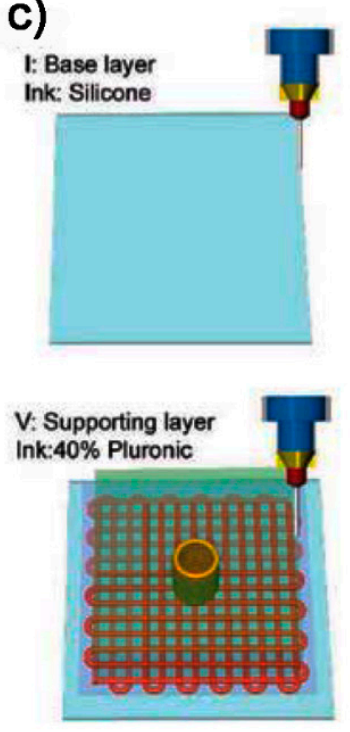

b)
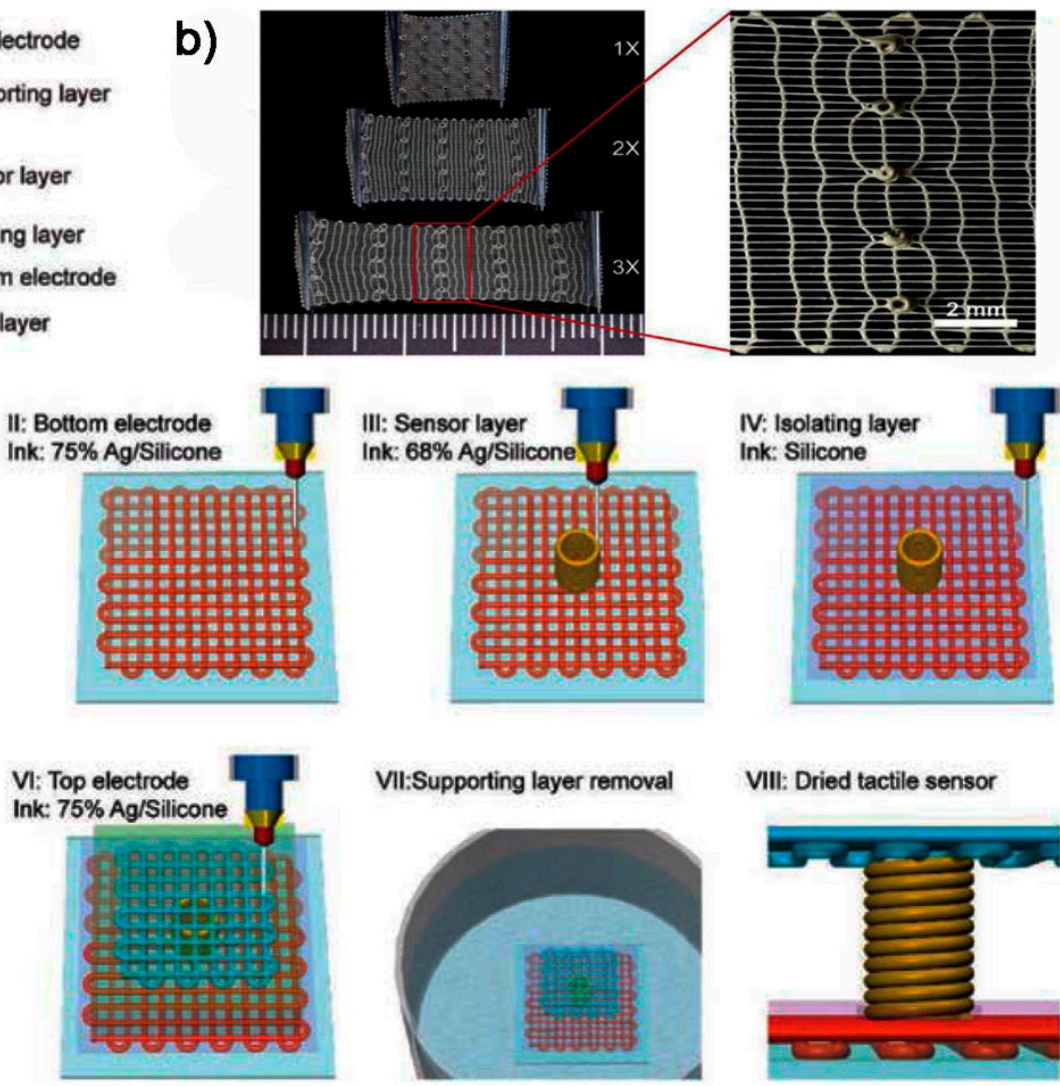

VIII: Dried tactile sensor

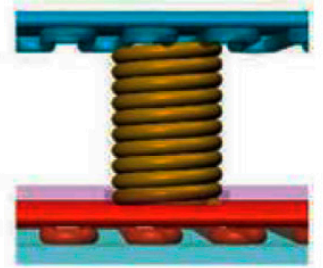

Figure 11. Tactile sensor design and 3D printing process: (a) schematic of the tactile sensor design; (b) stretchable tactile sensor fabricated via DIW; and (c) 3D printing procedures [141]. Reproduced with permission. Copyright John Wiley and Sons, 2017. 
Besides the commonly used materials prepared from the mixture of conductive fillers and elastomeric matrix, Kim et al. [142] developed a new carbon-based 3D printable composite dough materials for flexible sensors. The composite dough materials are comprised of an electrostatically assembled carbon composite and a thermoplastic triblock-copolymer elastomer, polystyrene-polyisoprene-polystyrene (SIS). To obtain a tailored spatial distribution of the conductive network, the authors modified multi-walled carbon nanotubes (MWCNTs) chemically to have surface-amine functional groups $\left(\mathrm{NH}_{2}-\mathrm{MWCNTs}\right)$. By introducing the surface-amine functional groups, nanotubes were confined to the surface of graphene oxides (GOs), thereby generating an electrostatically assembled carbon composite. Then the 3D printable dough materials were prepared by mixing the $\mathrm{NH}_{2}$-MWCNTs/GO composite fillers with SIS elastomer matrix. The authors firstly measured the piezoresistive response of GO-free MWCNTs/SIS composites. It was found that the use of MWCNTs alone was not appropriate for fabricating highly sensitive strain sensors, although excellent stretchability was achieved. By introducing GOs, only a slight amount of GOs incorporation $\left(\mathrm{NH}_{2}-\mathrm{MWCNTs} / \mathrm{GO}\right.$ ratio $\left.=450\right)$ resulted in a dramatic increase of gauge factor, however the linearity and hysteresis behavior were inferior. When $\mathrm{NH}_{2}$-MWCNTs/GO ratio was 9, a gauge factor of 72 was obtained. Meanwhile, excellent linearity of 0.94 and reduced hysteresis were achieved. By tuning the compositions of dough materials developed in this study, the sensor performance can be easily adjusted. It was also shown that the fabricated sensor was able to detect strains as low as $1 \%$.

DIW process also can be used to print liquid metals. For example, Boley et al. [143] developed a direct ink writing method to print gallium-indium (Ga-In) alloy on two types of substrates: glass and PDMS. It was found that the printed structures were able to maintain their shape due to the formation of gallium-oxide skin during the printing process. Strong adhesion between Ga-In alloy and substrate also facilitated the direct writing of Ga-In alloy. The authors directly printed Ga-In alloy onto PDMS substrate to obtain a strain sensor (Figure 12). The sensor showed good linearity between resistance change and strain (below 50\%) and a gauge factor of 1.5 was achieved.

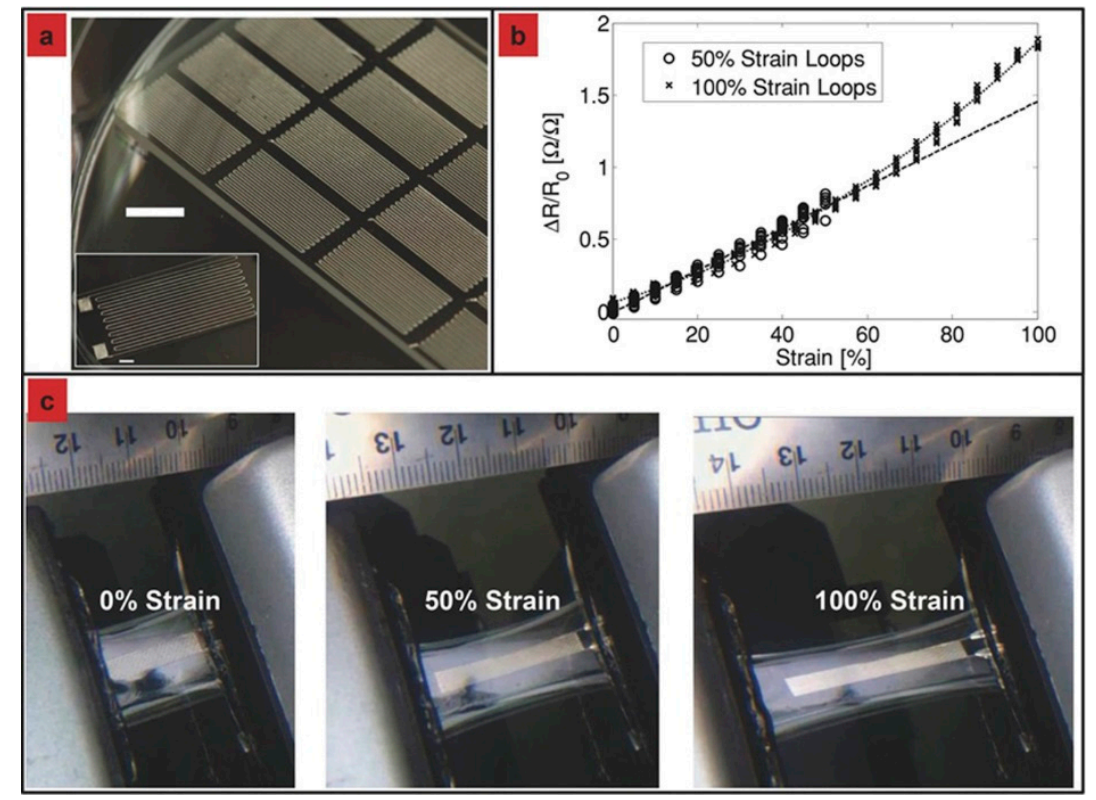

Figure 12. 3D-printed Ga-In alloy /PDMS strain sensor using DIW: (a) 3D-printed Ga-In alloy structures on glass; (b) resistance change with strain; and (c) strain sensor encapsulated in PDMS. Reproduced with permission. Copyright John Wiley and Sons, 2014.

Further, the research group developed a sequence of procedures toward all-printed flexible and stretchable tactile sensors [144]. The fabrication process (Figure 13a) included four steps: (i) printing of a base elastomer on the stage using DIW process; (ii) spray printing of liquid metal slurry (Ga-In 
alloy) using pressurized air; (iii) selective activation of the electrical path; and (iv) encapsulation by an elastomer to seal the device using DIW. During the spray printing of liquid metals, oxide shells formed around the deposited liquid particles. Therefore, the authors developed a selective activation process to rupture the oxide shells and merge the liquid particle cores to create an electrically conductive path by a pressure between the tapping stage and nozzle tip. It was demonstrated that printed electronics with complex geometries can be fabricated via this process. For example, the authors fabricated coil-shaped pressure sensor and serpentine-structured strain sensor with excellent stretchability and flexibility (as shown in Figure 13b-d). This new technology provides a feasible method for the fabrication of all-printed tactile sensors based on liquid metals.
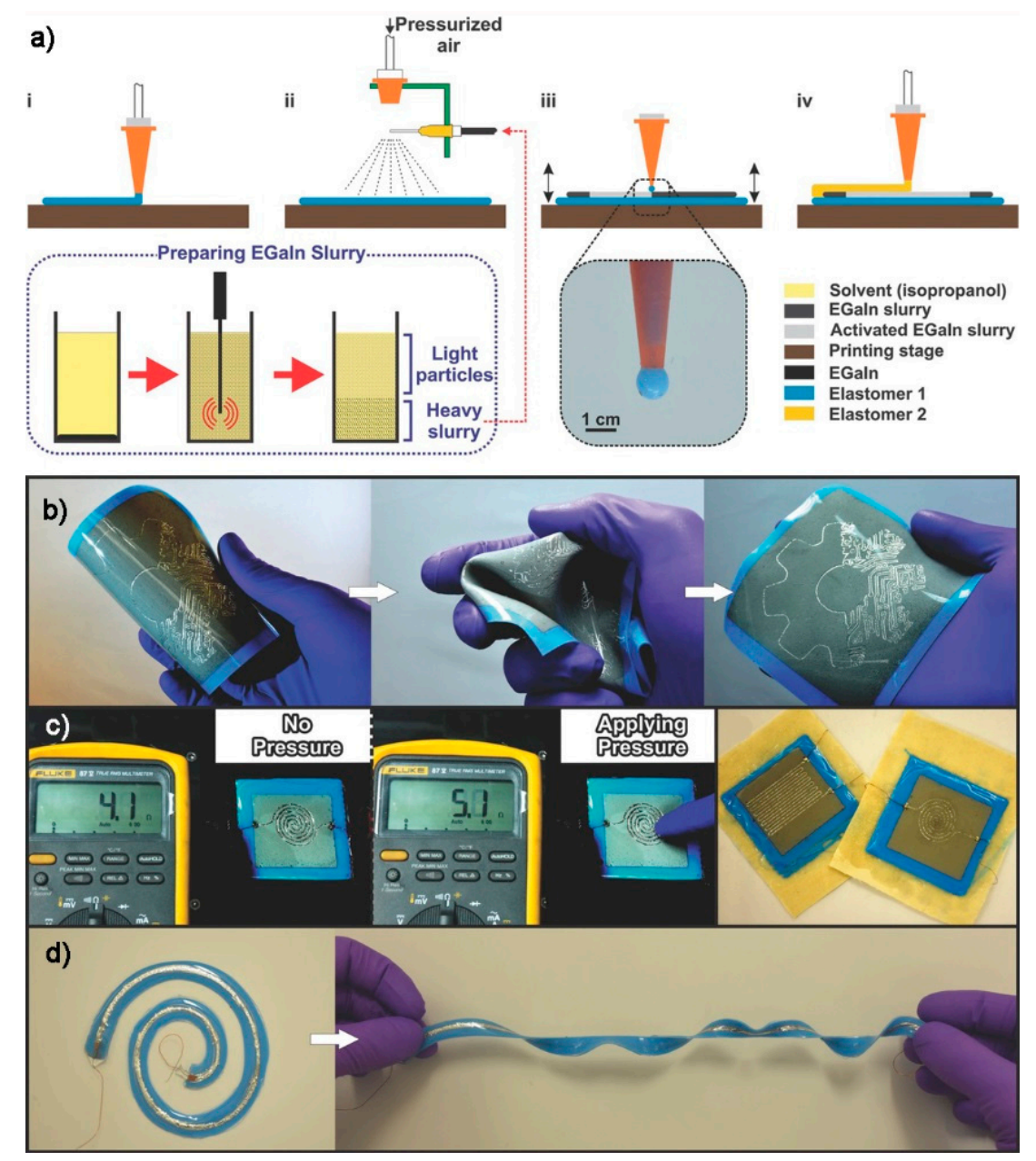

Figure 13. All-printed stretchable pressure and strain sensors based on liquid metals [144]: (a) the fabrication process; (b) printed complex electronics with excellent flexibility; (c) printed pressure and strain sensors; and (d) printed stretchable coil sensor. Reproduced with permission. Copyright John Wiley and Sons, 2017.

Conventional flexible tactile sensors are usually fabricated from organic elastomer matrices embedded with inorganic conductive materials and they usually detect signals through electronic conductors. In recent years, the concept of "ionic skin" using soft, biocompatible and ionically conductive hydrogels was proposed. The unique advantage of "ionic skin" is its biocompatibility with human tissue. Inspired by the idea of "ionic skin", Lei et al. [145] developed a capacitive multifunctional and flexible skin-like sensor by incorporating 3D printed thermo-responsive hydrogel into a capacitor circuit. The printed capacitive sensor was comprised of three layers, a polyethylene film as the dielectric layer sandwiched by two 3D-printed grid-structured thermo-responsive hydrogels as 
ionically conductive layer (Figure 14a,b). The capacitive sensor was able to output stable and sensitive capacitance-temperature response and also showed very high pressure sensitivity within $1 \mathrm{KPa}$. A pressure sensitivity of $0.45 \mathrm{KPa}^{-1}$ was achieved using the grid-structured hydrogel. The authors demonstrated its applications in detecting body temperature, gentle finger touches and finger bending motions. The combination of stimuli-responsive hydrogels and 3D printing may eventually lead to various multifunctional ionic sensors for wearable electronics.

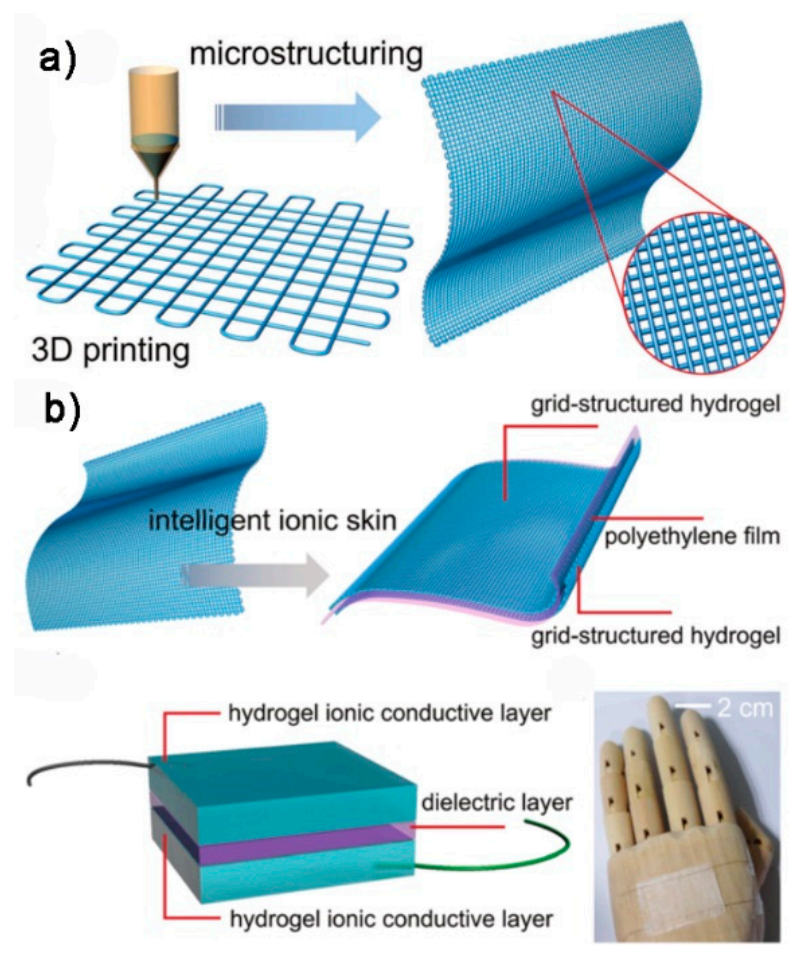

Figure 14. 3D-printed ionic tactile sensors: (a) 3D-printed grid-structured hydrogel film; and (b) tactile sensor structure and assembly [145]. Reproduced with permission. Copyright Royal Society of Chemistry, 2017.

Another example using stimuli-responsive hydrogels for 3D-printed flexible sensors was demonstrated by Liu et al. [146] They prepared a double-network hydrogel by combining an ionically cross-linked $k$-carrageenan network with a covalently cross-linked polyacrylamide (PAAm) network. It was found that the warm pregel solution of $k$-carrageenan/AAm had the required rheological properties for DIW. The printed structures have remarkable mechanical strength and self-healing properties after UV exposure. In addition, the hydrogel was also found to have good piezoresistive properties that is essential to strain sensors. The gauge factor of the double-network hydrogel was measured to be 0.23 at the strain of $100 \%$ and 0.63 at the strain of $1000 \%$. The authors also demonstrated the double-network hydrogel-based strain sensors in monitoring human finger bending motion.

In addition, DIW also can be used to print metal precursor solution on a stretchable substrate. After chemical reduction of precursor into metal nanoparticles, stretchable conductive electrodes with surface embedded metal particles can be obtained (Figure 15). Song et al. [147] developed a silver precursor (silver trifluoroacetate) solution and then the precursor solution was printed onto a poly(styrene- $b$-butadiene- $b$-styrene) (SBS) film substrate. A penetration process of precursor solution into the SBS substrate took place only a few seconds after printing. Then, a chemical reduction process by hydrazine vapor was applied to convert silver precursor into silver nanoparticles. It was found that highly conductive patterns with the thickness of $60 \mu \mathrm{m}$ could be easily obtained after repeated printing. The surface embedded conductive silver nanoparticles in the substrate can offer excellent stretchability and strong interfacial connections between conductive patterns and substrate. In comparison with 
conventional printing process, the interfacial connections between conductive materials and substrates are usually weak. The authors measured the resistance change of printed conductive line patterns at various strains. It was shown that the printed electrodes can not only be used as piezoresistive sensing element, but also stretchable, conformal and conductive electrodes.

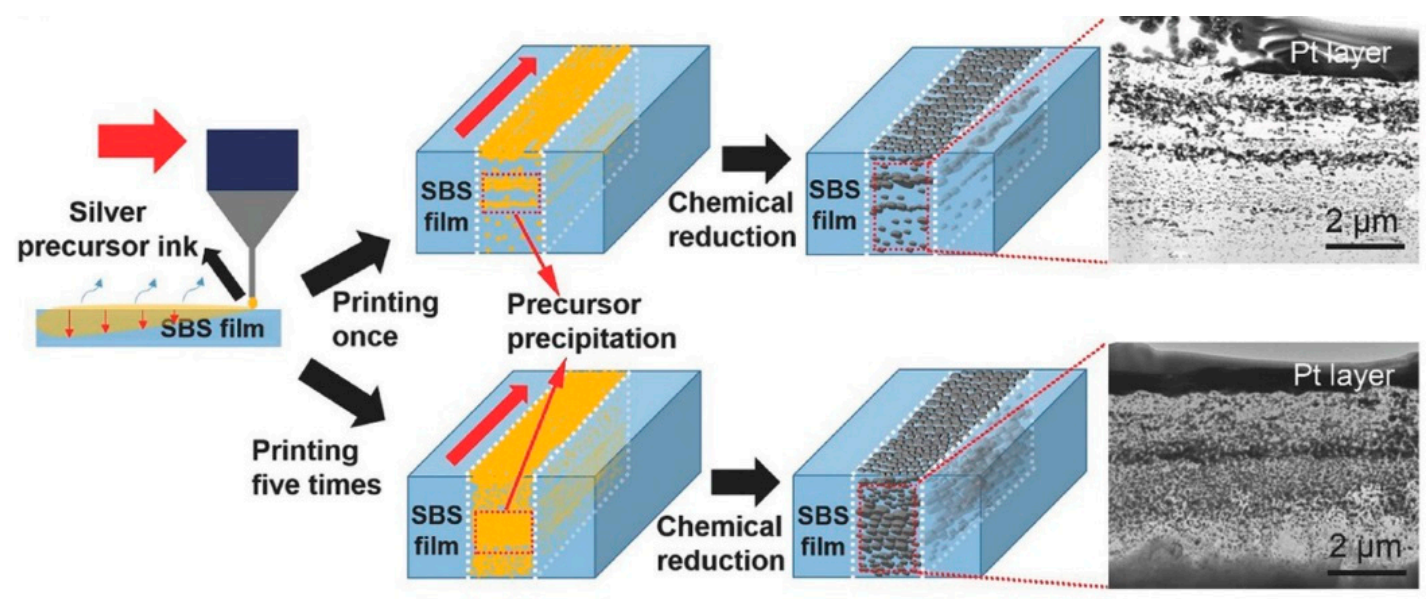

Figure 15. Printing metal precursor solution onto stretchable substrate to fabricate surface embedded conductive electrodes [147]. Reproduced with permission. Copyright John Wiley and Sons, 2017.

Silver nanoinks have also been used as printable inks for piezoresistive strain sensors. For example, Zhang et al. [148] printed silver nanoparticle-based inks on a polyurethane acrylate (PUA) substrate. Serpentine structured patterns were printed with different curvature radiuses. It was found that the stretchability and resistance change were strongly dependent on curvature radius. Serpentine structures with curvature radius of $200 \mu \mathrm{m}$ exhibited a record high gauge factor of $\sim 10^{7}$ at strain of $\sim 12 \%$. The increase of curvature radius resulted in higher stretchability and reduced gauge factor. The authors also demonstrated the printed strain sensors in detecting finger motion. Cai et al. [149] developed a printable silver nanowire-based ink that can be printed on various substrates including silicon wafer, PDMS and glass. The authors studied the variation of resistance change with different average nanowire lengths of 4.4, 15.6, $38.5 \mu \mathrm{m}$. It was found that longer nanowire length had better capability to maintain effective conductive network upon stretching. Meanwhile, smaller resistance change with applied strain was observed $(1000 \%$ resistance change for $4.4 \mu \mathrm{m}$ nanowire length and $90 \%$ for $38.5 \mu \mathrm{m}$ at the strain of $20 \%$ ). The silver nanowires on PDMS substrate can offer both excellent stretchability and conductive properties, therefore can be used as stretchable electrodes. The authors demonstrated a sandwich-structured capacitive pressure sensor composed of two printed stretchable electrodes and a silicone elastomer dielectric layer. The pressure sensor showed a pressure sensitivity of $0.106 \mathrm{KPa}^{-1}$ below $1 \mathrm{KPa}$ and a minimum detectable pressure of $\sim 100 \mathrm{~Pa}$.

In previously mentioned studies, various type of printable inks were developed to fabricate tactile sensors via DIW process including silicone/Ag composites, $\mathrm{NH}_{2}$-MWCNTs/GO/SIS composites, liquid metal-based inks, biocompatible hydrogels, metal precursor and silver nanoinks. It can be seen that DIW is a facile, simple and versatile technology with the fewest restrictions on printable materials. Meanwhile, DIW can also be used to fabricate tactile sensor components into tailored structures with tunable stretchability and electrical conductivity. For example, Wei et al. [150] printed free-standing wavy-structured stretchable electrodes based on a PDMS/MWCNTs composite ink. These electrodes were designed and fabricated with different structural parameters (Figure 16). The stretchability and resistance change upon repeated stretching were measured. It was found that different stretchability and resistance change properties were obtained for electrodes with different structural parameters. Through structural optimization, both high stretchability (300\%) and high resistance stability (5\% resistance change at $100 \%$ strain) can be achieved. 


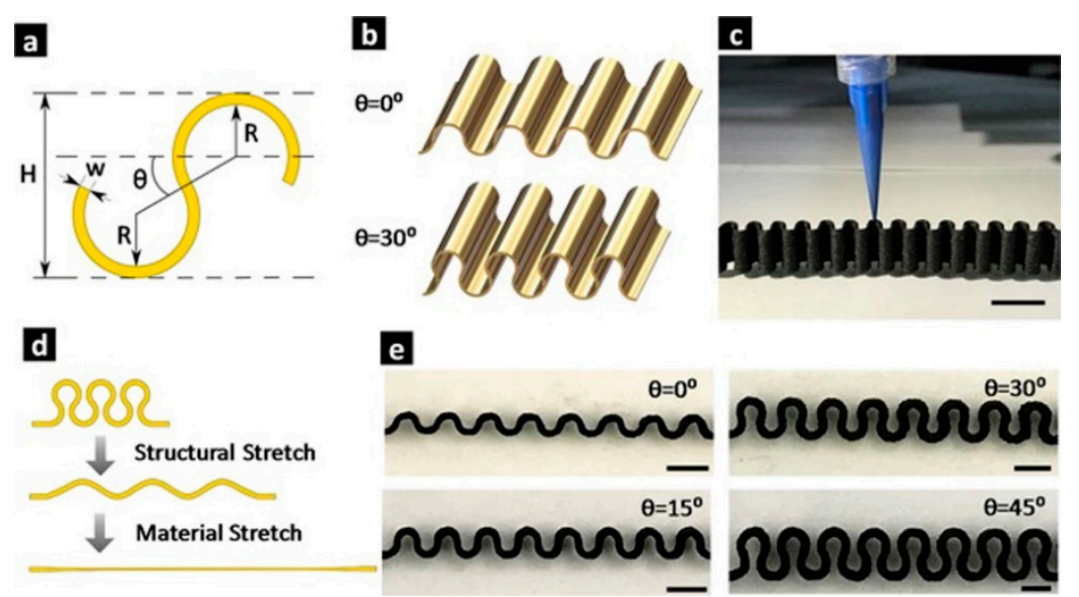

Figure 16. 3D-printed wavy-structured stretchable electrodes with different structural parameters. (a) Cross-sectional view of the serpentine design; (b) 3D schematics of the serpentine electrodes with $\theta=0^{\circ} / 30^{\circ}$ (fixed $R, w$ ); (c) photograph showing the 3D printing process. Scare bar $10 \mathrm{~mm}$; (d) schematics showing the unique two-degree stretching process of the serpentine design; (e) cross-sectional photographs of the 3D printed wavy electrodes with different joining angles. Scare bar $5 \mathrm{~mm}$. Reproduced from [150] with permission. Copyright John Wiley and Sons, 2017.

\section{(2) Embedded DIW for Tactile Sensors}

In the above DIW processes, the prepared inks were directly printed onto a flexible or stretchable substrate mounted on the building platform. Alternatively, inks can also be printed into a fluid medium reservoir and directly encapsulated in the fluid medium. Muth et al. [151] developed embedded DIW process to fabricate piezoresistive flexible strain sensors. In this process, conductive piezoresistive inks were directly printed within an elastomeric reservoir and encapsulated by a filler fluid (Figure 17a). Conductive carbon grease-a suspension of carbon black particles in silicone oil-was used as printable inks for the sensing element. Modified commercial silicone elastomer (uncured Ecoflex 00-30, Smooth-on Inc., Macungie, PA, USA) was used as the reservoir and filler fluid. After printing, the reservoir and filler fluid were co-cured to form a monolithic part with piezoresistive conductive ink encapsulated in the elastomeric matrix. Owing to the excellent stretchability of elastomeric matrix and conductive ink, the 3D-printed sensor could endure strains up to $700 \sim 800 \%$ mechanically and exhibit predictable electrical responses up to $\sim 400 \%$ strain (Figure $17 \mathrm{~b}$ ). The gauge factor of these flexible strain sensor was $3.8 \pm 0.6$ that was similar as conventional metallic strain gauges. The authors fabricated a glove with 3D-printed strain sensors encapsulated in elastomer for each finger that could be used to monitor the digit motion in real time. However, significant hysteresis was observed during cycling due to the inherent hysteresis associated with the elastomeric loading. 


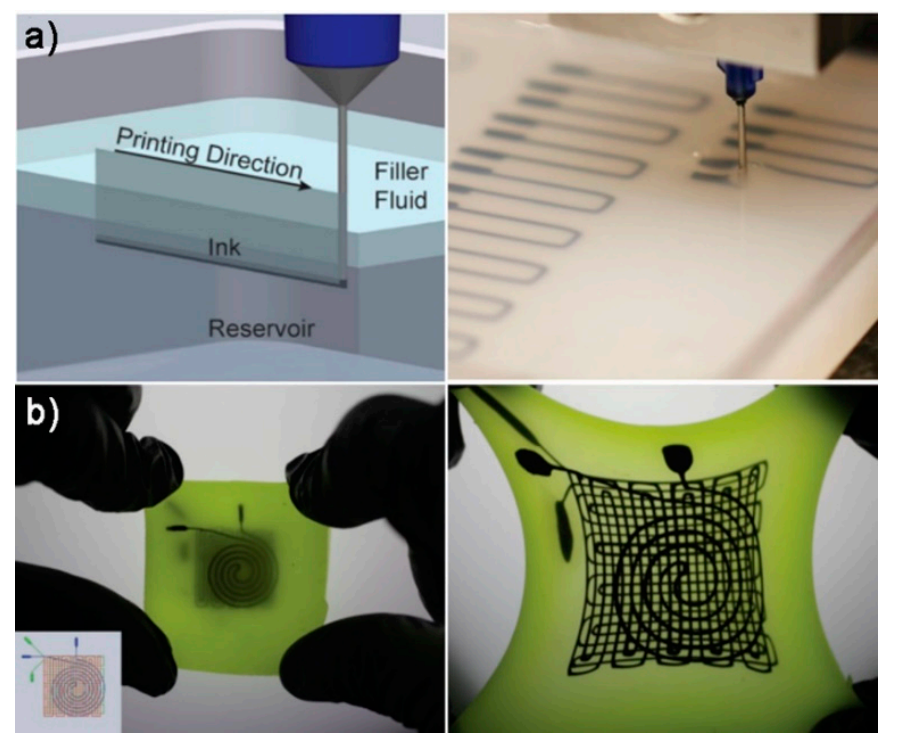

Figure 17. Embedded DIW process for tactile sensors: (a) embedded direct ink writing; and (b) fabrication of a flexible strain sensor in unstrained state and stretched state [151]. Reproduced with permission. Copyright John Wiley and Sons, 2017.

\section{(3) Multicore-Shell DIW for Tactile Sensors}

Another unique advantage of DIW process is its ability to enable the coextrusion of multiple inks to form multiple-layered fiber-shaped structures. For example, Frutiger et al. [152] developed a multicore-shell printing process to fabricate textile-mounted, capacitive flexible strain sensors. Ionically conductive ink composed of glycerol, sodium chloride, and polyethylene glycol was prepared and acted as the conductive layer. A commercially available silicone elastomer Dragonskin 10 was used as the dielectric layer. The fiber-shaped sensor consisted of four concentric layers including a core conductive fluid layer, dielectric silicone elastomer layer, an outer conductive fluid layer and an encapsulation silicone elastomer layer (Figure 18a,b). The four layers were coextruded through a customized printing head and deposited to form a capacitive fiber sensor. The authors measured the capacitance, resistance and decay time responses as a function of strain. It was demonstrated that the sensor could output any of these three quantities: capacitance, resistance and decay time. The gauge factor for the capacitive response of the sensor was $0.348 \pm 0.11$. The dynamic capacitance response was also measured for different strain amplitudes and frequencies. It was shown that the sensor output could accurately track the strain input. The authors also mounted the multicore-shell fiber sensor onto textiles across the knee by sewing and measured the decay time output for different walking speeds from 1 to $4 \mathrm{mph}$ (Figure 18c,d). It was demonstrated that the fiber sensor could be used to detect the walking gait. 

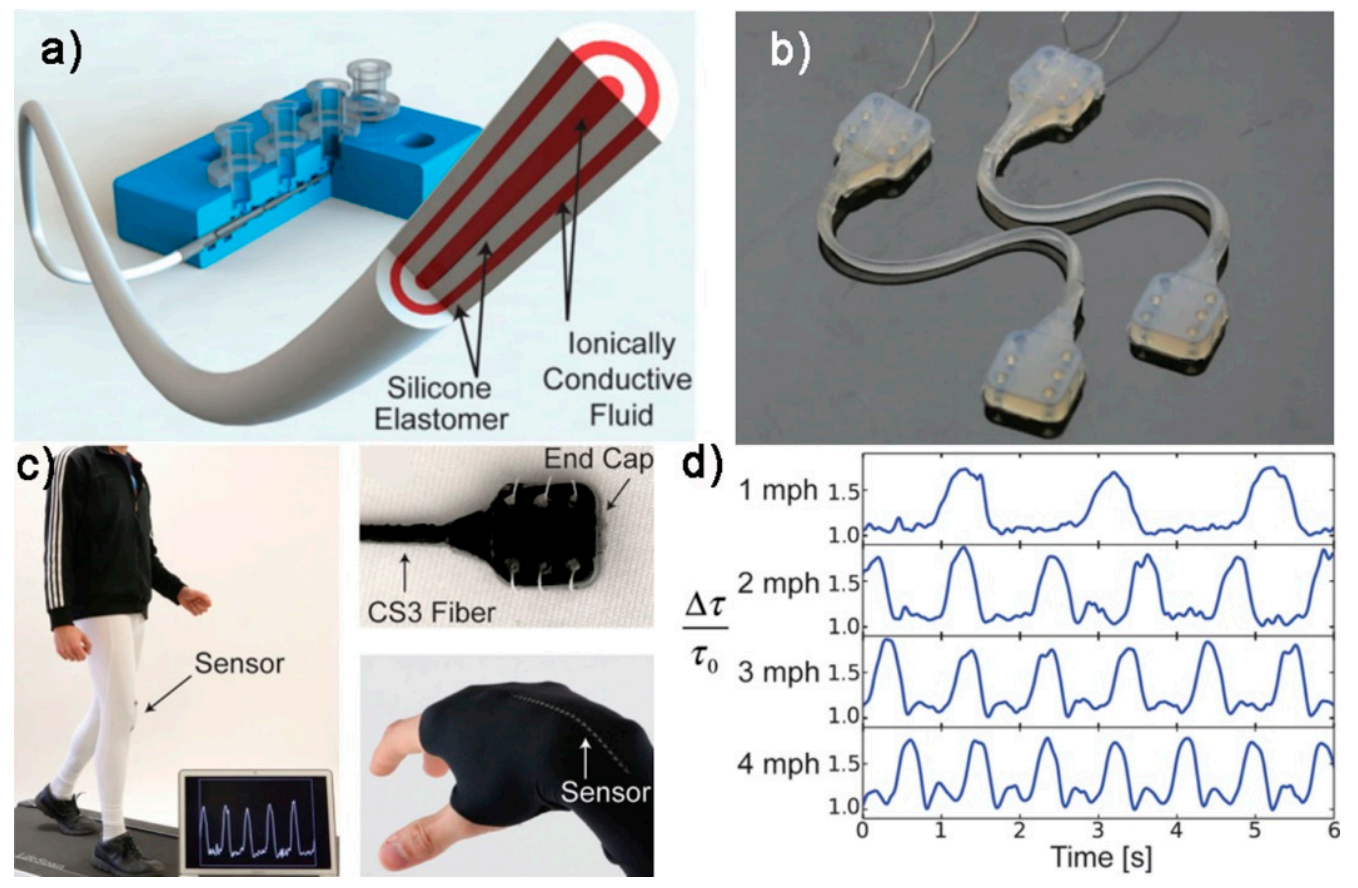

Figure 18. (a) Coextrusion of multiple inks to fabricate fiber-shaped multi-layered capacitive sensors; (b) the assembled sensor; (c) textile-mounted sensor for walking detection; and (d) the decay time response [152]. Reproduced with permission. Copyright John Wiley and Sons, 2017.

\subsection{Electrohydrodynamic 3D Printing for Piezoelectric Tactile Sensors}

EHD 3D printing is an emerging micro/nano fabrication technology based on electrostatic force-induced jetting flow of materials to fabricate micro/nanofibers. EHD 3D printing derives from a classical electrohydrodynamic flow jetting process: electrospinning which was developed as an important fiber production technology (Figure 19a). In this process, the polymer solution/melt jets generated from the Taylor cone undergo two stages. In the first stage, the polymer jets travel in a straight line and then goes through a "whipping process". Finally, the polymer jets elongate and deposit onto the building platform to form micro/nanofibers. Conventionally, the distance from nozzle tip to building platform was $5-20 \mathrm{~cm}$ and the inherent instability of electrospinning made the process controllability very poor. Using conventional electrospinning, nanofiber arrays of piezoelectric polymers such as PVDF and PVDF-TrFE can be fabricated and used as the piezoelectric sensing unit for tactile sensors. The applied high electrical fields for poling can greatly enhance the piezoelectric properties. However, the fiber alignment and orientation are difficult to control. Sun et al. proposed a near-field electrospinning (NFES) process that utilized the steady polymer jet region to achieve controlled deposition by reducing the distance between nozzle tip and building platform to $0.5-3 \mathrm{~mm}$ (Figure 19b). The modified process can be used to produce user specific patterns of micro/nanofibers in a simple and highly-efficient manner. By integrating the controlled deposition of NFES process and the layer-by-layer manufacturing principle of 3D printing, micro/nano 3D printing can be achieved. 

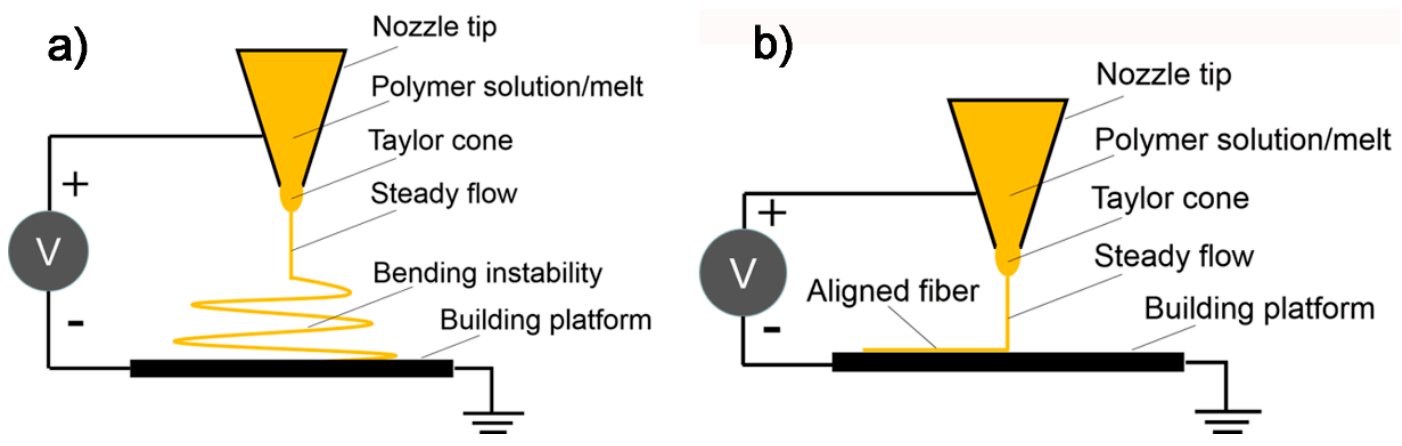

Figure 19. (a) Far-field electrospinning; and (b) near-field electrospinning.

Using EHD 3D printing, Fuh et al. [153] fabricated piezoelectric pressure sensors by depositing piezoelectric PVDF onto FDM-fabricated wavy substrates (Figure 20). Firstly, a thermoplastic elastomer substrate with topologically tailored wavy structures was fabricated via FDM process. Secondly, a copper foil was attached to the wavy substrate. Then, polyvinylidene fluoride (PVDF) micro/nano fibers were deposited onto the substrate surface by near-field electrospinning (NFES). Finally, the sensor was encapsulated by a PDMS film to form a piezoelectric pressure sensor. By using NFES, highly-aligned piezoelectric fiber array with diameter of $1-5 \mu \mathrm{m}$ was obtained. The authors fabricated three types of substrate including planar surface, square surface and sinusoidal surface structured surfaces. A total of 600 PVDF fibers were deposited and the output voltage was 2, 3.5 and $3.5 \mathrm{~V}$, respectively, for three types of wavy structured surfaces. The results showed that the sinusoidal surface had the best output, possibly due to the largest total length of PVDF fibers. The authors also demonstrated the application of the fabricated sensor in foot pressure measurement and human motion monitoring.

(a)

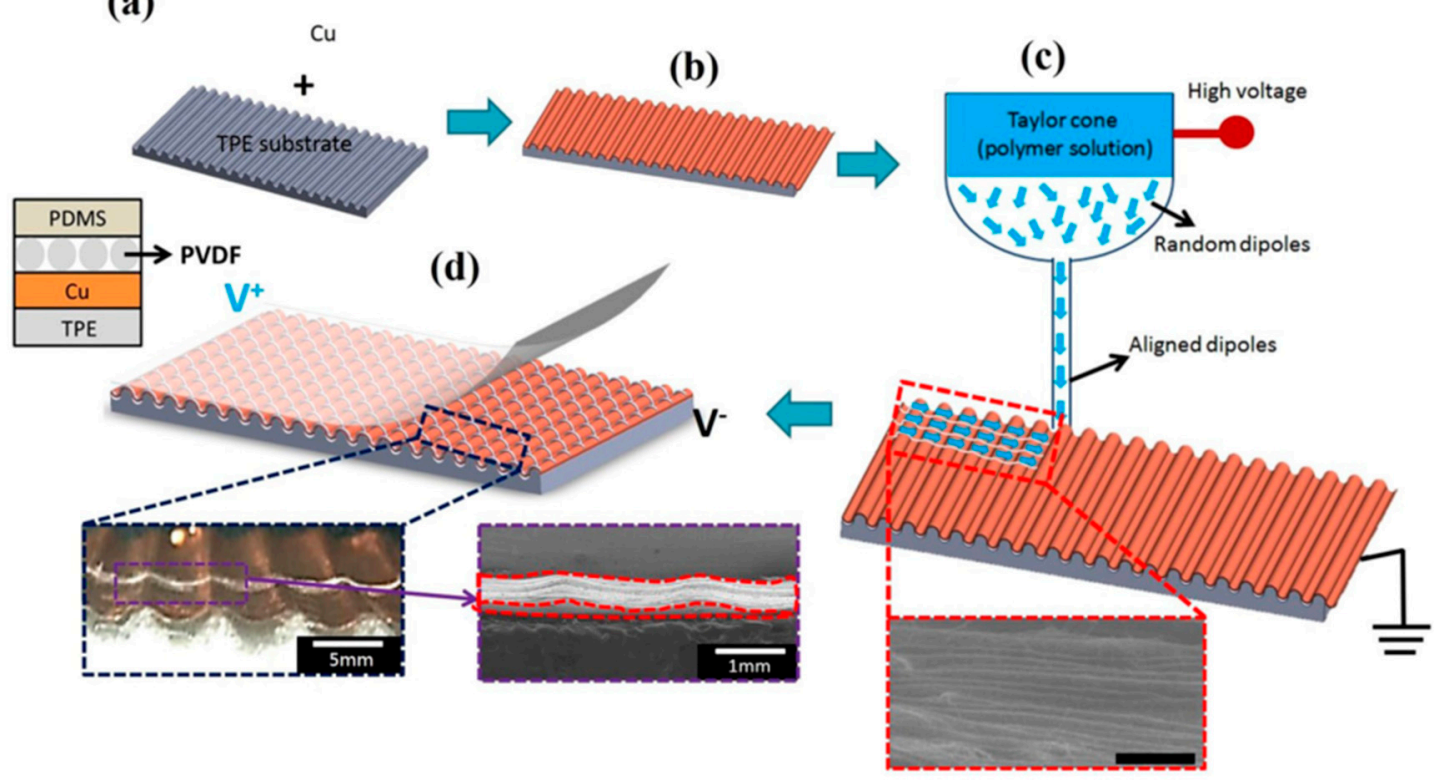

Figure 20. EHD printing of aligned PVDF micro/nanofibers on FDM-fabricated wavy structures: (a) FDM-fabricated TPE wavy substrate; (b) Cu foil adhesion on wavy substrate; (c) NFES of PVDF on wavy substrate; and (d) encapsulation with PDMS [153].

In addition to piezoelectric polymer, Qin et al. [154] used EHD 3D printing to fabricate coplanar capacitive touch sensors based on a composite ink composed of 20-30 wt \% Ag nanoparticles and triethylene glycol. The capacitive sensor was composed of two coplanar interdigitate microelectrode 
arrays printed on flexible PET film. The capacitance of the printed sensor was proportional to the number of electrodes. The authors measured the effects of finger touch on capacitance and it was demonstrated that the proposed sensor can be applied in touch displays.

\subsection{Comparison of Different 3D Printing Technologies for Tactile Sensors}

The previous section describes the $3 \mathrm{D}$ printing applications in tactile sensors. As for photopolymerization-based processes, the printable materials must be photocurable. Therefore, these processes are mainly limited to the fabrication of stretchable substrate and sensor body. Commercially available silicone rubber-like photopolymers can be directly used to fabricate flexible substrate and sensor body; however, they have limited stretchability. Elongation at break of $\sim 1100 \%$ that was comparable to the most stretchable silicone elastomer was achieved for recently developed UV curable elastomers. These progresses will finally lead to the development of 3D printable highly stretchable photopolymers with sufficient stretchability for tactile sensors. In addition, a unique advantage is the high precision and complexity that can be achieved through photopolymerization-based processes. However, available stretchable photopolymers are still very limited. With novel photocurable elastomeric materials being developed, photopolymerization-based processes may have wider applications in tactile sensors. For example, mixtures of stretchable photopolymers and conductive materials may be applied to the fabrication of stretchable electrodes and sensing element.

As for the FDM process, a unique advantage is its simplicity and low-cost. However, the printable materials must be processed into filaments. This has been a limitation for its applications in tactile sensors. To date, only a few types of materials are available for FDM. Thermoplastic elastomer such as TPU was used to fabricate wavy substrate and dielectric layer for tactile sensors. Composite materials of thermoplastic polymer and conductive filler such as PCL/CB and TPU/MWCNTs were also fabricated into piezoresistive sensing element by FDM. In addition, electric poling-assisted FDM process was developed to print piezoelectric polymers such PVDF into piezoelectric sensing element. However, the major limitation of this process is the limited stretchability of printable materials (failing strain of $60 \%$ for 5 wt $\%$ MWCNTs/TPU composites).

As for direct ink writing, by dispersing conductive fillers such as carbon black, MWCNTs, graphene, metal nanoparticles or nanowires into elastomeric matrices such as silicone elastomer, various type of printable composite inks can be obtained. By tuning the rheological properties, these printable inks can be used to fabricate stretchable substrate, electrodes and sensing element. Using multicore-shell printing and multi-material printing, fully 3D-printed tactile sensors can be achieved in a single printing procedure. An unrivaled advantage of DIW process is its wide range of printable materials and its capability to extrude silicone elastomer with the largest stretchability. In addition, printable ionic conductive hydrogels have also been demonstrated to fabricate ionic tactile sensors by DIW process. All these advantages will make DIW a competitive 3D printing technology for tactile sensor fabrication.

As for EHD 3D printing, NFES-based process has the capability of depositing piezoelectric polymer solution into micro/nanofibers with improved piezoelectric properties. This process provides a new way to fabricate highly-oriented micro/nanofiber arrays with excellent fiber alignment and may find important applications in piezoelectric tactile sensors. 
Table 1. 3D printing applications in tactile sensors.

\begin{tabular}{|c|c|c|c|c|c|c|}
\hline 3DP Technology & Sensing Mechanism & Loads & Printable Materials & Materials Shape & Printed Component & Ref. \\
\hline \multirow{3}{*}{ PolyJet } & Piezoresistive & Pressure & Photocurable TangoPlus & \multirow{4}{*}{$\begin{array}{l}\text { Photopolymer, } \\
\text { photopolymer } \\
\text { composites }\end{array}$} & Sensor body & {$[131]$} \\
\hline & Piezoresistive & Pressure & $\begin{array}{l}\text { Conductive photocurable } \\
\text { TangoPlus/MWCNTs composites }\end{array}$ & & Sensing element & [131] \\
\hline & Piezoresistive & Pressure & Photocurable VisiJet composites & & Sensor body & {$[132]$} \\
\hline DLP & - & - & UV curable EAA/AUD stretchable elastomer & & Substrate/Sensor body & {$[133]$} \\
\hline \multirow{6}{*}{ FDM } & Piezoresistive & Pressure & Conductive PCL/CB composite & \multirow{6}{*}{ Filament } & Sensing element & {$[134]$} \\
\hline & Piezoelectric & Pressure & Thermoplastic elastomer & & Sensor wavy substrate & [153] \\
\hline & Piezoresistive & Tensile strain & Conductive TPU/MWCNTs nanocomposites & & Sensing element & \\
\hline & Capacitive & Pressure & Thermoplastic elastomer & & Dielectric layer & [135] \\
\hline & Piezoresistive & Pressure & $\begin{array}{l}\text { Supporting structure: TPUSensing element: } \\
\text { TPU/MWCNTs composites }\end{array}$ & & $\begin{array}{l}\text { Supporting structure, sensing } \\
\text { element }\end{array}$ & [137] \\
\hline & Capacitive & Pressure & ABS & & $\begin{array}{l}\text { Molds for microstructuring } \\
\text { sensing element }\end{array}$ & [138] \\
\hline \multirow{3}{*}{$\begin{array}{c}\text { Electric } \\
\text { poling-assisted } \\
\text { FDM }\end{array}$} & Piezoelectric & Tensile strain & Piezoelectric PVDF polymer & Filament & Sensing element & [139] \\
\hline & Piezoelectric & Pressure & Piezoelectric PVDF polymer & Filament & Sensing element & {$[140]$} \\
\hline & Piezoelectric & Pressure & $\mathrm{PVDF} / \mathrm{BaTiO}_{3}$ composites & Filament & Sensing element & [140] \\
\hline \multirow{10}{*}{ Direct ink writing } & Piezoresistive & Pressure & $\begin{array}{l}\text { Sensing layer: } 68 \mathrm{wt} \% \mathrm{Ag} / \text { silicone ink } \\
\text { Electrode layer: } 75 \mathrm{wt} \% \mathrm{Ag} / \text { silicone ink } \\
\text { Substrate layer: silicone elastomer } \\
\text { Supporting layer: } 40 \% \text { pluronic ink }\end{array}$ & Inks & Fully 3D-printed tactile sensor & [141] \\
\hline & Piezoresistive & Tensile strain & $\begin{array}{l}\text { Composite dough materials: } \\
\mathrm{NH}_{2} \text {-MWCNTs/GO/SIS composites }\end{array}$ & Ink & Sensing element & [142] \\
\hline & Piezoresistive & Tensile strain & Gallium-indium alloy & Ink & Sensing element & {$[143]$} \\
\hline & Piezoresistive & $\begin{array}{l}\text { Pressure, tensile } \\
\text { strain }\end{array}$ & Elastomer, gallium-indium alloy & Ink & Fully 3D-printed tactile sensor & {$[144]$} \\
\hline & Capacitive & Pressure, temperature & Thermo-responsive hydrogel & Inks & Ionically conductive layer & [145] \\
\hline & Piezoresistive & Tensile strain & $\begin{array}{l}\text { K-carrageenan/PAAm double-network } \\
\text { hydrogel }\end{array}$ & Inks & Sensing element & [146] \\
\hline & Piezoresistive & Tensile strain & $\begin{array}{l}\text { Silver precursor (silver trifluoroacetate) } \\
\text { solution in alcohol or acetone }\end{array}$ & Inks & $\begin{array}{l}\text { Stretchable electrodes, sensing } \\
\text { element }\end{array}$ & [147] \\
\hline & - & Tensile strain & PDMS/MWCNTs composite & Inks & Stretchable electrodes & {$[150]$} \\
\hline & Piezoresistive & Tensile strain & Silver nanoparticle-based inks & Inks & Sensing element & [148] \\
\hline & Piezoresistive & $\begin{array}{l}\text { Tensile strain, } \\
\text { pressure }\end{array}$ & Silver nanowire-based inks & Inks & $\begin{array}{l}\text { Sensing element, stretchable } \\
\text { electrodes }\end{array}$ & [149] \\
\hline
\end{tabular}


Table 1. Cont.

\begin{tabular}{|c|c|c|c|c|c|c|}
\hline 3DP Technology & Sensing Mechanism & Loads & Printable Materials & Materials Shape & Printed Component & Ref. \\
\hline $\begin{array}{l}\text { Embedded direct ink } \\
\text { writing }\end{array}$ & Piezoresistive & Tensile strain & Suspensions of CB in silicone oil & Inks & Sensing element & {$[151$} \\
\hline $\begin{array}{l}\text { Multicore-shell direct } \\
\text { ink writing }\end{array}$ & Capacitive & Tensile strain & $\begin{array}{l}\text { Conductive layer: ionically conductive ink } \\
\text { composed of glycerol, NaCL, and PEG } \\
\text { Dielectric/encapsulation layer: modified } \\
\text { silicone elastomer }\end{array}$ & Inks & Fully 3D-printed tactile sensor & {$[152$} \\
\hline \multirow[b]{2}{*}{$\begin{array}{l}\text { electrohydrodynamic } \\
\text { printing }\end{array}$} & Piezoelectric & Pressure & Piezoelectric PVDF polymer & Polymer solution & Sensing element & {$[153$} \\
\hline & Capacitive & Finger touch & $\begin{array}{l}20 \mathrm{wt} \%-35 \mathrm{wt} \% \mathrm{Ag} \\
\text { nanoparticles/triethylene glycol composite }\end{array}$ & Composite solution & Conductive electrode & {$[154$} \\
\hline
\end{tabular}




\section{Conclusions and Outlook}

This review summarizes the latest advances in the fabrication of tactile sensors by 3D printing. In comparison with other fabrication methods such as lithography-based process, physical/chemical vapor deposition and various chemical processes, 3D printing can construct complex 3D geometries with customized topological features in a highly efficient and low-cost manner. Topologically optimized tactile components including substrate, electrodes and sensing element with tailored microstructures can be easily fabricated. Higher sensitivity, fast response, smaller minimal detectable loads and better flexibility can be achieved through structural optimization. This advantage will make 3D printing play a more important role in advancing wearable electronics and electronic skin. As for different types of 3D printing technologies, there are different requirements on printable materials. Photocurable polymers are required for photopolymerization-based 3D printing. Filament type materials are required for FDM process. The rheological properties of printable inks should be tuned for DIW process. These restrictions have limited the amounts of available printable materials. In the future, novel printable materials with improved stretchability need further development. In addition, some research groups have proposed multi-material 3D printing processes that can deposit different materials in one printing procedure to achieve fully 3D-printed tactile sensors. It can be expected that more multi-material printing technologies will emerge and these processes will greatly accelerate 3D printing applications in tactile sensors. Moreover, multifunctional tactile sensors that can detect not only force-related information but also temperature, humidity, etc. have been under development. In the future, it can be anticipated that 3D-printed multifunctional tactile sensor may become a research focus. Finally, inspired by the concept of ionic skin, 3D-printed ionic tactile sensors using biocompatible hydrogels also have good prospects in the future.

Author Contributions: C.L. (Changshi Lao) conceived and designed the structure of this review. C.L. (Changyong Liu) wrote the manuscript with the assistance of N.H., F.X. and J.T., Z.C., Y.F. and X.G. discussed and revised the manuscript.

Acknowledgments: This work was supported by the National Natural Science Foundation of China (No. 51705334), Guangdong Provincial Sci\&Tech Program (No. 2017B090911003), Natural Science Foundation of Shenzhen University (No. 2016034), Key Project Fund for Science and Technology Development of Guangdong Province (No. 2017B090911014) and Shenzhen Peacock Plan Technology Innovation Project (No. KQJSCX20170327150948772).

Conflicts of Interest: The authors declare no conflicts of interests.

\section{References}

1. Kim, D.H.; Lu, N.S.; Ma, R.; Kim, Y.S.; Kim, R.H.; Wang, S.D.; Wu, J.; Won, S.M.; Tao, H.; Islam, A.; et al. Epidermal electronics. Science 2011, 333, 838-843. [CrossRef] [PubMed]

2. Wang, S.D.; Li, M.; Wu, J.; Kim, D.H.; Lu, N.S.; Su, Y.W.; Kang, Z.; Huang, Y.G.; Rogers, J.A. Mechanics of epidermal electronics. J. Appl. Mech. Trans. ASME 2012, 79, 031022. [CrossRef]

3. Yeo, W.H.; Kim, Y.S.; Lee, J.; Ameen, A.; Shi, L.K.; Li, M.; Wang, S.D.; Ma, R.; Jin, S.H.; Kang, Z.; et al. Multifunctional epidermal electronics printed directly onto the skin. Adv. Mater. 2013, 25, 2773-2778. [CrossRef] [PubMed]

4. Wang, X.D.; Dong, L.; Zhang, H.L.; Yu, R.M.; Pan, C.F.; Wang, Z.L. Recent progress in electronic skin. Adv. Sci. 2015, 2, 1500169. [CrossRef] [PubMed]

5. Choi, S.; Lee, H.; Ghaffari, R.; Hyeon, T.; Kim, D.H. Recent advances in flexible and stretchable bio-electronic devices integrated with nanomaterials. Adv. Mater. 2016, 28, 4203-4218. [CrossRef] [PubMed]

6. Chortos, A.; Liu, J.; Bao, Z.A. Pursuing prosthetic electronic skin. Nat. Mater. 2016, 15, 937-950. [CrossRef] [PubMed]

7. Rim, Y.S.; Bae, S.H.; Chen, H.J.; De Marco, N.; Yang, Y. Recent progress in materials and devices toward printable and flexible Sensors. Adv. Mater. 2016, 28, 4415-4440. [CrossRef] [PubMed]

8. Han, S.T.; Peng, H.Y.; Sun, Q.J.; Venkatesh, S.; Chung, K.S.; Lau, S.C.; Zhou, Y.; Roy, V.A.L. An overview of the development of flexible sensors. Adv. Mater. 2017, 29, 1700375. [CrossRef] [PubMed] 
9. Wang, X.W.; Liu, Z.; Zhang, T. Flexible sensing electronics for wearable/attachable health monitoring. Small 2017, 13, 1602790. [CrossRef] [PubMed]

10. Yang, T.T.; Xie, D.; Li, Z.H.; Zhu, H.W. Recent advances in wearable tactile sensors: Materials, sensing mechanisms, and device performance. Mater. Sci. Eng. R 2017, 115, 1-37. [CrossRef]

11. Chen, S.; Jiang, K.; Lou, Z.; Chen, D.; Shen, G.Z. Recent developments in graphene-based tactile sensors and e-skins. Adv. Mater. Technol. 2018, 3, 1700248. [CrossRef]

12. Zou, Z.N.; Zhu, C.P.; Li, Y.; Lei, X.F.; Zhang, W.; Xiao, J.L. Rehealable, fully recyclable, and malleable electronic skin enabled by dynamic covalent thermoset nanocomposite. Sci. Adv. 2018, 4, eaaq0508. [CrossRef] [PubMed]

13. Amjadi, M.; Kyung, K.U.; Park, I.; Sitti, M. Stretchable, skin-mountable, and wearable strain sensors and their potential applications: A review. Adv. Funct. Mater. 2016, 26, 1678-1698. [CrossRef]

14. Heikenfeld, J.; Jajack, A.; Rogers, J.; Gutruf, P.; Tian, L.; Pan, T.; Li, R.; Khine, M.; Kim, J.; Wang, J.; et al. Wearable sensors: Modalities, challenges, and prospects. Lab Chip 2018, 18, 217-248. [CrossRef] [PubMed]

15. Yao, S.S.; Swetha, P.; Zhu, Y. Nanomaterial-enabled wearable sensors for healthcare. Adv. Healthc. Mater. 2018, 7, 1700889. [CrossRef] [PubMed]

16. Pang, C.; Lee, G.Y.; Kim, T.I.; Kim, S.M.; Kim, H.N.; Ahn, S.H.; Suh, K.Y. A flexible and highly sensitive strain-gauge sensor using reversible interlocking of nanofibres. Nat. Mater. 2012, 11, 795-801. [CrossRef] [PubMed]

17. Obitayo, W.; Liu, T. A Review: Carbon nanotube-based piezoresistive strain sensors. J. Sens. 2012, 2012, 652438. [CrossRef]

18. Yamada, T.; Hayamizu, Y.; Yamamoto, Y.; Yomogida, Y.; Izadi-Najafabadi, A.; Futaba, D.N.; Hata, K. A stretchable carbon nanotube strain sensor for human-motion detection. Nat. Nanotechnol. 2011, 6, $296-301$. [CrossRef] [PubMed]

19. Hu, N.; Fukunaga, H.; Atobe, S.; Liu, Y.; Li, J. Piezoresistive strain sensors made from carbon nanotubes based polymer nanocomposites. Sensors 2011, 11, 10691-10723.

20. Eswaraiah, V.; Balasubramaniam, K.; Ramaprabhu, S. Functionalized graphene reinforced thermoplastic nanocomposites as strain sensors in structural health monitoring. J. Mater. Chem. 2011, 21, 12626. [CrossRef]

21. Li, X.; Zhang, R.J.; Yu, W.J.; Wang, K.L.; Wei, J.Q.; Wu, D.H.; Cao, A.Y.; Li, Z.H.; Cheng, Y.; Zheng, Q.H.; et al. Stretchable and highly sensitive graphene-on-polymer strain sensors. Sci. Rep. 2012, 2, 870. [CrossRef] [PubMed]

22. Yao, H.B.; Ge, J.; Wang, C.F.; Wang, X.; Hu, W.; Zheng, Z.J.; Ni, Y.; Yu, S.H. A flexible and highly pressure-sensitive graphene-polyurethane sponge based on fractured microstructure design. Adv. Mater. 2013, 25, 6692-6698. [CrossRef] [PubMed]

23. Amjadi, M.; Pichitpajongkit, A.; Lee, S.; Ryu, S.; Park, I. Highly stretchable and sensitive strain sensor based on silver nanowire-elastomer nanocomposite. ACS Nano 2014, 8, 5154-5163. [CrossRef] [PubMed]

24. Choong, C.L.; Shim, M.B.; Lee, B.S.; Jeon, S.; Ko, D.S.; Kang, T.H.; Bae, J.; Lee, S.H.; Byun, K.E.; Im, J.; et al. Highly stretchable resistive pressure sensors using a conductive elastomeric composite on a micropyramid array. Adv. Mater. 2014, 26, 3451-3458. [CrossRef] [PubMed]

25. Chun, S.; Kim, Y.; Jin, H.; Choi, E.; Lee, S.-B.; Park, W. A graphene force sensor with pressure-amplifying structure. Carbon 2014, 78, 601-608. [CrossRef]

26. Gong, S.; Schwalb, W.; Wang, Y.W.; Chen, Y.; Tang, Y.; Si, J.; Shirinzadeh, B.; Cheng, W.L. A wearable and highly sensitive pressure sensor with ultrathin gold nanowires. Nat. Commun. 2014, 5, 3132. [CrossRef] [PubMed]

27. Hou, C.; Wang, H.; Zhang, Q.; Li, Y.; Zhu, M. Highly conductive, flexible, and compressible all-graphene passive electronic skin for sensing human touch. Adv. Mater. 2014, 26, 5018-5024. [CrossRef] [PubMed]

28. Park, Y.J.; Lee, S.K.; Kim, M.S.; Kim, H.; Ahn, J.H. Graphene-based conformal devices. ACS Nano 2014, 8, 7655-7662. [CrossRef] [PubMed]

29. Viry, L.; Levi, A.; Totaro, M.; Mondini, A.; Mattoli, V.; Mazzolai, B.; Beccai, L. Flexible three-axial force sensor for soft and highly sensitive artificial touch. Adv. Mater. 2014, 26, 2659-2664. [CrossRef] [PubMed]

30. Yan, C.; Wang, J.; Kang, W.; Cui, M.; Wang, X.; Foo, C.Y.; Chee, K.J.; Lee, P.S. Highly stretchable piezoresistive graphene-nanocellulose nanopaper for strain sensors. Adv. Mater. 2014, 26, 2022-2027. [CrossRef] [PubMed] 
31. Yang, T.T.; Wang, Y.; Li, X.M.; Zhang, Y.Y.; Li, X.; Wang, K.L.; Wu, D.H.; Jin, H.; Li, Z.H.; Zhu, H.W. Torsion sensors of high sensitivity and wide dynamic range based on a graphene woven structure. Nanoscale 2014, 6 , 13053-13059. [CrossRef] [PubMed]

32. Chun, S.; Kim, Y.; Oh, H.S.; Bae, G.; Park, W. A highly sensitive pressure sensor using a double-layered graphene structure for tactile sensing. Nanoscale 2015, 7, 11652-11659. [CrossRef] [PubMed]

33. He, Z.F.; Chen, W.J.; Liang, B.H.; Liu, C.Y.; Yang, L.L.; Lu, D.W.; Mo, Z.C.; Zhu, H.; Tang, Z.K.; Gui, X.C. Capacitive pressure sensor with high sensitivity and fast response to dynamic interaction based on graphene and porous nylon networks. ACS Appl. Mater. Inter. 2018, 10, 12816-12823. [CrossRef] [PubMed]

34. Kim, J.; Lee, M.S.; Jeon, S.; Kim, M.; Kim, S.; Kim, K.; Bien, F.; Hong, S.Y.; Park, J.U. Highly transparent and stretchable field-effect transistor sensors using graphene-nanowire hybrid nanostructures. Adv. Mater. 2015, 27, 3292-3297. [CrossRef] [PubMed]

35. Kim, S.Y.; Park, S.; Park, H.W.; Park, D.H.; Jeong, Y.; Kim, D.H. Highly sensitive and multimodal all-carbon skin sensors capable of simultaneously detecting tactile and biological stimuli. Adv. Mater. 2015, 27, 4178-4185. [CrossRef] [PubMed]

36. Liu, Q.; Zhang, M.; Huang, L.; Li, Y.R.; Chen, J.; Li, C.; Shi, G.Q. High-quality graphene ribbons prepared from graphene xxide hydrogels and their application for strain sensors. ACS Nano 2015, 9, 12320-12326. [CrossRef] [PubMed]

37. Rahimi, R.; Ochoa, M.; Yu, W.Y.; Ziaie, B. Highly stretchable and sensitive unidirectional strain sensor via laser carbonization. ACS Appl. Mater. Interfaces 2015, 7, 4463-4470. [CrossRef] [PubMed]

38. Tian, H.; Shu, Y.; Wang, X.F.; Mohammad, M.A.; Bie, Z.; Xie, Q.Y.; Li, C.; Mi, W.T.; Yang, Y.; Ren, T.L. A graphene-based resistive pressure sensor with record-high sensitivity in a wide pressure range. Sci. Rep. 2015, 5, 8603. [CrossRef] [PubMed]

39. Yang, T.T.; Wang, W.; Zhang, H.Z.; Li, X.M.; Shi, J.D.; He, Y.J.; Zheng, Q.S.; Li, Z.H.; Zhu, H.W. Tactile sensing system based on arrays of graphene woven microfabrics: Electromechanical behavior and electronic skin application. ACS Nano 2015, 9, 10867-10875. [CrossRef] [PubMed]

40. Lai, Y.C.; Ye, B.W.; Lu, C.F.; Chen, C.T.; Jao, M.H.; Su, W.F.; Hung, W.Y.; Lin, T.Y.; Chen, Y.F. Extraordinarily sensitive and low-voltage operational cloth-based electronic skin for wearable sensing and multifunctional integration uses: A tactile-induced insulating-to-conducting transition. Adv. Funct. Mater. 2016, 26, 1286-1295. [CrossRef]

41. Li, J.H.; Zhao, S.F.; Zeng, X.L.; Huang, W.P.; Gong, Z.Y.; Zhang, G.P.; Sun, R.; Wong, C.P. Highly stretchable and sensitive strain sensor based on facilely prepared three-dimensional graphene foam composite. ACS Appl. Mater. Interfaces 2016, 8, 18954-18961. [CrossRef] [PubMed]

42. Lou, Z.; Chen, S.; Wang, L.; Jiang, K.; Shen, G. An ultra-sensitive and rapid response speed graphene pressure sensors for electronic skin and health monitoring. Nano Energy 2016, 23, 7-14. [CrossRef]

43. Shi, G.; Zhao, Z.H.; Pai, J.H.; Lee, I.; Zhang, L.Q.; Stevenson, C.; Ishara, K.; Zhang, R.J.; Zhu, H.W.; Ma, J. Highly sensitive, wearable, durable strain sensors and stretchable conductors using graphene/silicon rubber composites. Adv. Funct. Mater. 2016, 26, 7614-7625. [CrossRef]

44. Shi, J.; Li, X.; Cheng, H.; Liu, Z.; Zhao, L.; Yang, T.; Dai, Z.H.; Cheng, Z.G.; Shi, E.Z.; Yang, L.; et al. Graphene reinforced carbon nanotube networks for wearable strain sensors. Adv. Funct. Mater. 2016, 26, 2078-2084. [CrossRef]

45. Chen, Q.; Xu, R.; He, Z.; Zhao, K.; Pan, L. Printing 3D gel polymer electrolyte in lithium-ion microbattery using stereolithography. J. Electrochem. Soc. 2017, 164, A1852-A1857. [CrossRef]

46. Foster, C.W.; Down, M.P.; Zhang, Y.; Ji, X.; Rowley-Neale, S.J.; Smith, G.C.; Kelly, P.J.; Banks, C.E. 3D printed graphene based energy storage devices. Sci. Rep. 2017, 7, 42233. [CrossRef] [PubMed]

47. Fu, K.; Wang, Y.B.; Yan, C.Y.; Yao, Y.G.; Chen, Y.A.; Dai, J.Q.; Lacey, S.; Wang, Y.B.; Wan, J.Y.; Li, T.; et al. Graphene oxide-based electrode inks for 3D-printed lithium-ion batteries. Adv. Mater. 2016, 28, 2587-2594. [CrossRef] [PubMed]

48. Hu, J.T.; Jiang, Y.; Cui, S.H.; Duan, Y.D.; Liu, T.C.; Guo, H.; Lin, L.P.; Lin, Y.; Zheng, J.X.; Amine, K.; et al. 3D-printed cathodes of $\mathrm{LiMn}_{1-\mathrm{x}} \mathrm{Fe}_{\mathrm{x}} \mathrm{PO}_{4}$ nanocrystals achieve both ultrahigh rate and high capacity for advanced lithium-ion battery. Adv. Energy Mater. 2016, 6, 1600856. [CrossRef]

49. Kohlmeyer, R.R.; Blake, A.J.; Hardin, J.O.; Carmona, E.A.; Carpena-Nunez, J.; Maruyama, B.; Berrigan, J.D.; Huang, H.; Durstock, M.F. Composite batteries: A simple yet universal approach to 3D printable lithium-ion battery electrodes. J. Mater. Chem. A 2016, 4, 16856-16864. [CrossRef] 
50. Li, J.; Leu, M.C.; Panat, R.; Park, J. A hybrid three-dimensionally structured electrode for lithium-ion batteries via 3D printing. Mater. Des. 2017, 119, 417-424. [CrossRef]

51. Liu, C.; Cheng, X.; Li, B.; Chen, Z.; Mi, S.; Lao, C. Fabrication and characterization of 3D-printed highly-porous 3D LiFePO4 electrodes by low temperature direct writing process. Materials 2017, 10, 934. [CrossRef] [PubMed]

52. Rocha, V.G.; Garcia-Tunon, E.; Botas, C.; Markoulidis, F.; Feilden, E.; D’Elia, E.; Ni, N.; Shaffer, M.; Saiz, E. Multimaterial 3D printing of graphene-based electrodes for electrochemical energy storage using thermoresponsive inks. ACS Appl. Mater. Interfaces 2017, 9, 37136-37145. [CrossRef] [PubMed]

53. Sun, K.; Wei, T.S.; Ahn, B.Y.; Seo, J.Y.; Dillon, S.J.; Lewis, J.A. 3D printing of interdigitated Li-ion microbattery architectures. Adv. Mater. 2013, 25, 4539-4543. [CrossRef] [PubMed]

54. Wang, Y.; Chen, C.; Xie, H.; Gao, T.; Yao, Y.; Pastel, G.; Han, X.G.; Li, Y.J.; Zhao, J.P.; Fu, K.K.; et al. 3D-printed all-fiber Li-ion battery toward wearable energy storage. Adv. Funct. Mater. 2017, 27, 1703140. [CrossRef]

55. Areir, M.; Xu, Y.M.; Harrison, D.; Fyson, J. 3D printing of highly flexible supercapacitor designed for wearable energy storage. Mater. Sci. Eng. B 2017, 226, 29-38. [CrossRef]

56. Huang, W.H.; Finnerty, C.; Sharp, R.; Wang, K.; Balili, B. High-performance 3D printed microtubular solid oxide fuel cells. Adv. Mater. Technol. 2017, 2, 1600258. [CrossRef]

57. Philamore, H.; Rossiter, J.; Walters, P.; Winfield, J.; Ieropoulos, I. Cast and 3D printed ion exchange membranes for monolithic microbial fuel cell fabrication. J. Power Sources 2015, 289, 91-99. [CrossRef]

58. Wang, Q.; Yu, Y.; Yang, J.; Liu, J. Fast fabrication of flexible functional circuits based on liquid metal dual-trans printing. Adv. Mater. 2015, 27, 7109-7116. [CrossRef] [PubMed]

59. Wu, S.Y.; Yang, C.; Hsu, W.; Lin, L. 3D-printed microelectronics for integrated circuitry and passive wireless sensors. Microsyst. Nanoeng. 2015, 1, 15013-15021. [CrossRef]

60. Zheng, Y.; He, Z.; Gao, Y.; Liu, J. Direct desktop printed-circuits-on-paper flexible electronics. Sci. Rep. 2013, 3, 1786. [CrossRef]

61. Liu, C.Y.; Li, Y.; Zhang, L.; Mi, S.L.; Xu, Y.Y.; Sun, W. Development of a novel low-temperature deposition machine using screw extrusion to fabricate poly(L-lactide-co-glycolide) acid scaffolds. Proc. Inst. Mech. Eng. H 2014, 228, 593-606. [CrossRef] [PubMed]

62. Xu, Y.Y.; Guo, X.; Yang, S.T.; Li, L.; Zhang, P.; Sun, W.; Liu, C.Y.; Mi, S.L. Construction of bionic tissue engineering cartilage scaffold based on three-dimensional printing and oriented frozen technology. J. Biomed. Mater. Res. A 2018, 106, 1664-1676. [CrossRef] [PubMed]

63. Wu, Z.J.; Su, X.; Xu, Y.Y.; Kong, B.; Sun, W.; Mi, S.L. Bioprinting three-dimensional cell-laden tissue constructs with controllable degradation. Sci. Rep. 2016, 6, 24474. [CrossRef] [PubMed]

64. Kong, B.; Sun, W.; Chen, G.S.; Tang, S.; Li, M.; Shao, Z.W.; Mi, S.L. Tissue-engineered cornea constructed with compressed collagen and laser-perforated electrospun mat. Sci. Rep. 2017, 7, 970. [CrossRef] [PubMed]

65. Xu, Y.Y.; Wu, X.Y.; Guo, X.; Kong, B.; Zhang, M.; Qian, X.; Mi, S.L.; Sun, W. The boom in 3D-printed sensor technology. Sensors 2017, 17, 1166. [CrossRef] [PubMed]

66. Chun, S.; Hong, A.; Choi, Y.; Ha, C.; Park, W. A tactile sensor using a conductive graphene-sponge composite. Nanoscale 2016, 8, 9185-9192. [CrossRef] [PubMed]

67. Someya, T.; Sekitani, T.; Iba, S.; Kato, Y.; Kawaguchi, H.; Sakurai, T. A large-area, flexible pressure sensor matrix with organic field-effect transistors for artificial skin applications. Proc. Natl. Acad. Sci. USA 2004, 101, 9966-9970. [CrossRef] [PubMed]

68. Tok, J.B.H.; Bao, Z.A. Recent advances in flexible and stretchable electronics, sensors and power sources. Sci. China Chem. 2012, 55, 718-725. [CrossRef]

69. Chortos, A.; Bao, Z.N. Skin-inspired electronic devices. Mater. Today 2014, 17, 321-331. [CrossRef]

70. Lee, Y.; Oh, J.Y.; Kim, T.R.; Gu, X.D.; Kim, Y.; Wang, G.J.N.; Wu, H.-C.; Pfattner, R.; To, J.W.F.; Katsumata, T.; et al. Deformable organic nanowire field-effect transistors. Adv. Mater. 2018, 30, 1704401. [CrossRef] [PubMed]

71. Wang, G.J.N.; Gasperini, A.; Bao, Z.A. Stretchable polymer semiconductors for plastic electronics. Adv. Electron. Mater. 2018, 4, 1700429. [CrossRef]

72. Wang, S.H.; Xu, J.; Wang, W.C.; Wang, G.J.N.; Rastak, R.; Molina-Lopez, F.; Chung, J.W.; Niu, S.M.; Feig, V.R.; Lopez, J.; et al. Skin electronics from scalable fabrication of an intrinsically stretchable transistor array. Nature 2018, 555, 83-88. [CrossRef] [PubMed] 
73. Kim, K.K.; Hong, S.; Cho, H.M.; Lee, J.; Suh, Y.D.; Ham, J.; Ko, S.H. Highly sensitive and stretchable multidimensional strain sensor with prestrained anisotropic metal nanowire percolation networks. Nano Lett. 2015, 15, 5240-5247. [CrossRef] [PubMed]

74. Zhang, S.J.; Zhang, H.L.; Yao, G.; Liao, F.Y.; Gao, M.; Huang, Z.L.; Li, K.Y.; Lin, Y. Highly stretchable, sensitive, and flexible strain sensors based on silver nanoparticles/carbon nanotubes composites. J. Alloy. Compd. 2015, 652, 48-54. [CrossRef]

75. Zhang, X.; Hu, S.; Wang, M.; Yu, J.; Khan, Q.; Shang, J.; Ba, L. Continuous graphene and carbon nanotube based high flexible and transparent pressure sensor arrays. Nanotechnology 2015, 26, 115501. [CrossRef] [PubMed]

76. Yu, G.H.; Hu, J.D.; Tan, J.P.; Gao, Y.; Lu, Y.F.; Xuan, F.Z. A wearable pressure sensor based on ultraviolet/ozone microstructured carbon nanotube/polydimethylsiloxane arrays for electronic skins. Nanotechnology 2018, 29, 115502. [CrossRef] [PubMed]

77. Amjadi, M.; Yoon, Y.J.; Park, I. Ultra-stretchable and skin-mountable strain sensors using carbon nanotubes-Ecoflex nanocomposites. Nanotechnology 2015, 26, 375501. [CrossRef] [PubMed]

78. Cho, D.; Park, J.; Kim, J.; Kim, T.; Kim, J.; Park, I.; Jeon, S. Three-dimensional continuous conductive nanostructure for highly sensitive and stretchable strain sensor. ACS Appl. Mater. Interfaces 2017, 9, 17370-17379. [CrossRef] [PubMed]

79. Lee, J.; Lim, M.; Yoon, J.; Kim, M.S.; Choi, B.; Kim, D.M.; Kim, D.H.; Park, I.; Choi, S. Transparent, flexible strain sensor based on a solution-processed carbon nanotube network. ACS Appl. Mater. Interfaces 2017, 9, 26279-26285. [CrossRef] [PubMed]

80. Park, S.; Vosguerichian, M.; Bao, Z.A. A review of fabrication and applications of carbon nanotube film-based flexible electronics. Nanoscale 2013, 5, 1727-1752. [CrossRef] [PubMed]

81. Pan, L.J.; Chortos, A.; Yu, G.H.; Wang, Y.Q.; Isaacson, S.; Allen, R.; Shi, Y.; Dauskardt, R.; Bao, Z.N. An ultra-sensitive resistive pressure sensor based on hollow-sphere microstructure induced elasticity in conducting polymer film. Nat. Commun. 2014, 5, 3002. [CrossRef] [PubMed]

82. Yang, Z.; Wang, D.Y.; Pang, Y.; Li, Y.X.; Wang, Q.; Zhang, T.Y.; Wang, J.B.; Liu, X.; Yang, Y.Y.; Jian, J.M.; et al. Simultaneously detecting subtle and intensive human motions based on a silver nanoparticles bridged graphene strain sensor. ACS Appl. Mater. Interfaces 2018, 10, 3948-3954. [CrossRef] [PubMed]

83. Lee, J.; Kim, S.; Lee, J.; Yang, D.; Park, B.C.; Ryu, S.; Park, I. A stretchable strain sensor based on a metal nanoparticle thin film for human motion detection. Nanoscale 2014, 6, 11932-11939. [CrossRef] [PubMed]

84. Tee, B.C.K.; Wang, C.; Allen, R.; Bao, Z.N. An electrically and mechanically self-healing composite with pressure- and flexion-sensitive properties for electronic skin applications. Nat. Nanotechnol. 2012, 7, 825-832. [CrossRef] [PubMed]

85. Bae, S.-H.; Lee, Y.; Sharma, B.K.; Lee, H.-J.; Kim, J.-H.; Ahn, J.-H. Graphene-based transparent strain sensor. Carbon 2013, 51, 236-242. [CrossRef]

86. Kwon, O.K.; Lee, J.H.; Kim, K.-S.; Kang, J.W. Developing ultrasensitive pressure sensor based on graphene nanoribbon: Molecular dynamics simulation. Phys. E Low-Dimens. Syst. Nanostruct. 2013, 47, 6-11. [CrossRef]

87. Zhu, S.-E.; Krishna Ghatkesar, M.; Zhang, C.; Janssen, G.C.A.M. Graphene based piezoresistive pressure sensor. Appl. Phys. Lett. 2013, 102, 161904. [CrossRef]

88. Gonçalves, V.; Brandão, L.; Mendes, A. Development of porous polymer pressure sensors incorporating graphene platelets. Polym. Test. 2014, 37, 129-137. [CrossRef]

89. Jeong, Y.R.; Park, H.; Jin, S.W.; Hong, S.Y.; Lee, S.-S.; Ha, J.S. Highly stretchable and sensitive strain sensors using fragmentized graphene foam. Adv. Funct. Mater. 2015, 25, 4228-4236. [CrossRef]

90. Kazemzadeh, R.; Andersen, K.; Motha, L.; Kim, W.S. Highly sensitive pressure sensor array with photothermally reduced graphene oxide. IEEE Electron Device Lett. 2015, 36, 180-182. [CrossRef]

91. Samad, Y.A.; Li, Y.Q.; Schiffer, A.; Alhassan, S.M.; Liao, K. Graphene foam developed with a novel two-step technique for low and high strains and pressure-sensing applications. Small 2015, 11, 2380-2385. [CrossRef] [PubMed]

92. Wang, W.; Yang, T.; Zhu, H.; Zheng, Q. Bio-inspired mechanics of highly sensitive stretchable graphene strain sensors. Appl. Phys. Lett. 2015, 106, 171903. [CrossRef]

93. Yeo, J.C.; Yu, J.; Shang, M.; Loh, K.P.; Lim, C.T. Highly flexible graphene oxide nanosuspension liquid-based microfluidic tactile sensor. Small 2016, 12, 1593-1604. 
94. Lin, Y.; Liu, S.Q.; Chen, S.; Wei, Y.; Dong, X.C.; Liu, L. A highly stretchable and sensitive strain sensor based on graphene-elastomer composites with a novel double-interconnected network. J. Mater. Chem. C 2016, 4, 6345-6352. [CrossRef]

95. Tadakaluru, S.; Kumpika, T.; Kantarak, E.; Sroila, W.; Panthawan, A.; Sanmuangmoon, P.; Thongsuwan, W.; Singjai, P. Highly stretchable and sensitive strain sensors using nano-graphene coated natural rubber. Plast. Rubber Compos. 2017, 46, 301-305. [CrossRef]

96. Akdogan, E.K.; Allahverdi, M.; Safari, A. Piezoelectric composites for sensor and actuator applications. IEEE Ultrason. Ferroelectr. 2005, 52, 746-775. [CrossRef]

97. Gullapalli, H.; Vemuru, V.S.M.; Kumar, A.; Botello-Mendez, A.; Vajtai, R.; Terrones, M.; Nagarajaiah, S.; Ajayan, P.M. Flexible piezoelectric ZnO-paper nanocomposite strain sensor. Small 2010, 6, 1641-1646. [CrossRef] [PubMed]

98. Liang, R.J.; Wang, Q.M. High sensitivity piezoelectric sensors using flexible PZT thick-film for shock tube pressure testing. Sens. Actuators A Phys. 2015, 235, 317-327. [CrossRef]

99. Chen, Z.F.; Wang, Z.; Li, X.M.; Lin, Y.X.; Luo, N.Q.; Long, M.Z.; Zhao, N.; Xu, J.B. Flexible piezoelectric-induced pressure sensors for static measurements based on nanowires/graphene heterostructures. ACS Nano 2017, 11, 4507-4513. [CrossRef] [PubMed]

100. Liu, Z.; Zhang, S.; Jin, Y.M.; Ouyang, H.; Zou, Y.; Wang, X.X.; Xie, L.X.; Li, Z. Flexible piezoelectric nanogenerator in wearable self-powered active sensor for respiration and healthcare monitoring. Semicond. Sci. Technol. 2017, 32, 064004. [CrossRef]

101. Chen, S.W.; Wu, N.; Ma, L.; Lin, S.Z.; Yuan, F.; Xu, Z.S.; Li, W.B.; Wang, B.; Zhou, J. Noncontact heartbeat and respiration monitoring based on a hollow microstructured self-powered pressure sensor. ACS Appl. Mater. Interfaces 2018, 10, 3660-3667. [CrossRef] [PubMed]

102. Persano, L.; Dagdeviren, C.; Su, Y.W.; Zhang, Y.H.; Girardo, S.; Pisignano, D.; Huang, Y.G.; Rogers, J.A. High performance piezoelectric devices based on aligned arrays of nanofibers of poly(vinylidenefluoride-co-trifluoroethylene). Nat. Commun. 2013, 4, 1633. [CrossRef] [PubMed]

103. Park, S.H.; Lee, H.B.; Yeon, S.M.; Park, J.; Lee, N.K. Flexible and stretchable piezoelectric sensor with thickness-tunable configuration of electrospun nanofiber mat and elastomeric substrates. ACS Appl. Mater. Interfaces 2016, 8, 24773-24781. [CrossRef] [PubMed]

104. Xing, L.D.; Zhu, R.J.; Wang, Z.M.; Wang, F.X.; Kimura, H. Flexible tensile strain sensor based on lead-free 0.5Ba $\left(\mathrm{Ti}_{0.8} \mathrm{Zr}_{0.2}\right) \mathrm{O}_{3}-0.5\left(\mathrm{Ba}_{0.7} \mathrm{Ca}_{0.3}\right) \mathrm{TiO}_{3}$ piezoelectric nanofibers. Smart Mater. Struct. 2017, 26, [CrossRef]

105. Deutz, D.B.; Mascarenhas, N.T.; Schelen, J.B.J.; de Leeuw, D.M.; van der Zwaag, S.; Groen, P. Flexible piezoelectric touch sensor by alignment of lead-free alkaline niobate microcubes in PDMS. Adv. Funct. Mater. 2017, 27, 1700728. [CrossRef]

106. Mannsfeld, S.C.B.; Tee, B.C.K.; Stoltenberg, R.M.; Chen, C.V.H.H.; Barman, S.; Muir, B.V.O.; Sokolov, A.N.; Reese, C.; Bao, Z.N. Highly sensitive flexible pressure sensors with microstructured rubber dielectric layers. Nat. Mater. 2010, 9, 859-864. [CrossRef] [PubMed]

107. Lipomi, D.J.; Vosgueritchian, M.; Tee, B.C.K.; Hellstrom, S.L.; Lee, J.A.; Fox, C.H.; Bao, Z.N. Skin-like pressure and strain sensors based on transparent elastic films of carbon nanotubes. Nat. Nanotechnol. 2011, 6, 788-792. [CrossRef] [PubMed]

108. Cohen, D.J.; Mitra, D.; Peterson, K.; Maharbiz, M.M. A highly elastic, capacitive strain gauge based on percolating nanotube networks. Nano Lett. 2012, 12, 1821-1825. [CrossRef] [PubMed]

109. Tee, B.C.K.; Chortos, A.; Dunn, R.R.; Schwartz, G.; Eason, E.; Bao, Z.A. Tunable flexible pressure sensors using microstructured elastomer geometries for intuitive electronics. Adv. Funct. Mater. 2014, 24, 5427-5434. [CrossRef]

110. Kwon, D.; Lee, T.I.; Shim, J.; Ryu, S.; Kim, M.S.; Kim, S.; Kim, T.S.; Park, I. Highly sensitive, flexible, and wearable pressure sensor based on a giant piezocapacitive effect of three-dimensional microporous elastomeric dielectric layer. ACS Appl. Mater. Interfaces 2016, 8, 16922-16931. [CrossRef] [PubMed]

111. Li, T.; Luo, H.; Qin, L.; Wang, X.W.; Xiong, Z.P.; Ding, H.Y.; Gu, Y.; Liu, Z.; Zhang, T. Flexible capacitive tactile sensor based on micropatterned dielectric layer. Small 2016, 12, 5042-5048. [CrossRef] [PubMed]

112. Chhetry, A.; Yoon, H.; Park, J.Y. A flexible and highly sensitive capacitive pressure sensor based on conductive fibers with a microporous dielectric for wearable electronics. J. Mater. Chem. C 2017, 5, 10068-10076. [CrossRef] 
113. Kang, M.; Kim, J.; Jang, B.; Chae, Y.; Kim, J.H.; Ahn, J.H. Graphene-based three-dimensional capacitive touch sensor for wearable electronics. ACS Nano 2017, 11, 7950-7957. [CrossRef] [PubMed]

114. Atalay, O.; Atalay, A.; Gafford, J.; Walsh, C. A highly sensitive capacitive-based soft pressure sensor based on a conductive fabric and a microporous dielectric layer. Adv. Mater. Technol. 2018, 3, 1700237. [CrossRef]

115. Cheng, W.; Wang, J.; Ma, Z.; Yan, K.; Wang, Y.M.; Wang, H.T.; Li, S.; Li, Y.; Pan, L.J.; Shi, Y. Flexible pressure sensor with high sensitivity and low hysteresis based on a hierarchically microstructured electrode. IEEE Electron Device Lett. 2018, 39, 288-291. [CrossRef]

116. Lin, M.F.; Xiong, J.Q.; Wang, J.X.; Parida, K.; Lee, P.S. Core-shell nanofiber mats for tactile pressure sensor and nanogenerator applications. Nano Energy 2018, 44, 248-255. [CrossRef]

117. Park, S.; Kim, H.; Vosgueritchian, M.; Cheon, S.; Kim, H.; Koo, J.H.; Kim, T.R.; Lee, S.; Schwartz, G.; Chang, H.; et al. Stretchable energy-harvesting tactile electronic skin capable of differentiating multiple mechanical stimuli modes. Adv. Mater. 2014, 26, 7324-7332. [CrossRef] [PubMed]

118. Boutry, C.M.; Nguyen, A.; Lawal, Q.O.; Chortos, A.; Rondeau-Gagne, S.; Bao, Z.N. A sensitive and biodegradable pressure sensor array for cardiovascular monitoring. Adv. Mater. 2015, 27, 6954-6961. [CrossRef] [PubMed]

119. Frazier, W.E. Metal additive manufacturing: A review. J. Mater. Eng. Perform. 2014, 23, 1917-1928. [CrossRef]

120. Herzog, D.; Seyda, V.; Wycisk, E.; Emmelmann, C. Additive manufacturing of metals. Acta Mater. 2016, 117, 371-392. [CrossRef]

121. Schmidt, M.; Merklein, M.; Bourell, D.; Dimitrov, D.; Hausotte, T.; Wegener, K.; Overmeyer, L.; Vollertsen, F.; Levy, G.N. Laser based additive manufacturing in industry and academia. CIRP Ann. 2017, 66, 561-583. [CrossRef]

122. Durmus, N.G.; Tasoglu, S.; Demirci, U. Bioprinting functional droplet networks. Nat. Mater. 2013, 12, 478-479. [CrossRef] [PubMed]

123. Mironov, V.; Prestwich, G.; Forgacs, G. Bioprinting living structures. J. Mater. Chem. 2007, 17, $2054-2060$. [CrossRef]

124. Norotte, C.; Marga, F.S.; Niklason, L.E.; Forgacs, G. Scaffold-free vascular tissue engineering using bioprinting. Biomaterials 2009, 30, 5910-5917. [CrossRef] [PubMed]

125. Rodriguez-Devora, J.I.; Zhang, B.M.; Reyna, D.; Shi, Z.D.; Xu, T. High throughput miniature drug-screening platform using bioprinting technology. Biofabrication 2012, 4, 035001. [CrossRef] [PubMed]

126. Tasoglu, S.; Demirci, U. Bioprinting for stem cell research. Trends Biotechnol. 2013, 31, 10-19. [CrossRef] [PubMed]

127. Au, A.K.; Huynh, W.; Horowitz, L.F.; Folch, A. 3D-printed microfluidics. Angew. Chem. Int. Ed. 2016, 55, 3862-3881. [CrossRef] [PubMed]

128. Bhattacharjee, N.; Urrios, A.; Kanga, S.; Folch, A. The upcoming 3D-printing revolution in microfluidics. Lab Chip 2016, 16, 1720-1742. [CrossRef] [PubMed]

129. Ho, C.M.B.; Ng, S.H.; Li, K.H.H.; Yoon, Y.J. 3D printed microfluidics for biological applications. Lab Chip 2015, 15, 3627-3637. [CrossRef] [PubMed]

130. Zhang, B.; He, J.K.; Li, X.; Xu, F.Y.; Li, D.C. Micro/nanoscale electrohydrodynamic printing: From 2D to 3D. Nanoscale 2016, 8, 15376-15388. [CrossRef] [PubMed]

131. Vatani, M.; Lu, Y.; Engeberg, E.D.; Choi, J.-W. Combined 3D printing technologies and material for fabrication of tactile sensors. Int. J. Precis. Eng. Manuf. 2015, 16, 1375-1383. [CrossRef]

132. Agarwala, S.; Goh, G.L.; Yap, Y.L.; Goh, G.D.; Yu, H.; Yeong, W.Y.; Tran, T. Development of bendable strain sensor with embedded microchannels using 3D printing. Sens. Actuators A Phys. 2017, 263, 593-599. [CrossRef]

133. Patel, D.K.; Sakhaei, A.H.; Layani, M.; Zhang, B.; Ge, Q.; Magdassi, S. Highly stretchable and UV curable elastomers for digital light processing based 3D printing. Adv. Mater. 2017, 29, 1606000. [CrossRef] [PubMed]

134. Leigh, S.J.; Bradley, R.J.; Purssell, C.P.; Billson, D.R.; Hutchins, D.A. A simple, low-cost conductive composite material for 3D printing of electronic sensors. PLoS ONE 2012, 7, e49365. [CrossRef] [PubMed]

135. Saari, M.; Xia, B.; Cox, B.; Krueger, P.S.; Cohen, A.L.; Richer, E. Fabrication and analysis of a composite 3D printed capacitive force sensor. 3D Print. Add. Manuf. 2016, 3, 136-141. [CrossRef]

136. Christ, J.F.; Aliheidari, N.; Ameli, A.; Potschke, P. 3D printed highly elastic strain sensors of multiwalled carbon nanotube/thermoplastic polyurethane nanocomposites. Mater. Des. 2017, 131, 394-401. [CrossRef] 
137. Kim, K.; Park, J.; Suh, J.H.; Kim, M.; Jeong, Y.; Park, I. 3D printing of multiaxial force sensors using carbon nanotube (CNT)/thermoplastic polyurethane (TPU) filaments. Sens. Actuators A Phys. 2017, 263, 493-500. [CrossRef]

138. Zhuo, B.G.; Chen, S.J.; Zhao, M.M.; Guo, X.J. High sensitivity flexible capacitive pressure sensor using polydimethylsiloxane elastomer dielectric layer micro-structured by $3 \mathrm{D}$ printed mold. IEEE J. Electron Devices Soc. 2017, 5, 219-223. [CrossRef]

139. Lee, C.; Tarbutton, J.A. Electric poling-assisted additive manufacturing process for PVDF polymer-based piezoelectric device applications. Smart Mater. Struct. 2014, 23, 095044. [CrossRef]

140. Kim, H.; Torres, F.; Wu, Y.; Villagran, D.; Lin, Y.; Tseng, T.-L. Integrated 3D printing and corona poling process of PVDF piezoelectric films for pressure sensor application. Smart Mater. Struct. 2017, 26, 085027. [CrossRef]

141. Guo, S.Z.; Qiu, K.Y.; Meng, F.B.; Park, S.H.; McAlpine, M.C. 3D printed stretchable tactile sensors. Adv. Mater. 2017, 29, 1701218. [CrossRef] [PubMed]

142. Kim, J.Y.; Ji, S.; Jung, S.; Ryu, B.H.; Kim, H.S.; Lee, S.S.; Choi, Y.; Jeong, S. 3D printable composite dough for stretchable, ultrasensitive and body-patchable strain sensors. Nanoscale 2017, 9, 11035-11046. [CrossRef] [PubMed]

143. Boley, J.W.; White, E.L.; Chiu, G.T.C.; Kramer, R.K. Direct writing of gallium-indium alloy for stretchable electronics. Adv. Funct. Mater. 2014, 24, 3501-3507. [CrossRef]

144. Mohammed, M.G.; Kramer, R. All-printed flexible and stretchable electronics. Adv. Mater. 2017, $29,1604965$. [CrossRef] [PubMed]

145. Lei, Z.Y.; Wang, Q.K.; Wu, P.Y. A multifunctional skin-like sensor based on a 3D printed thermo-responsive hydrogel. Mater. Horiz. 2017, 4, 694-700. [CrossRef]

146. Liu, S.J.; Li, L. Ultrastretchable and self-healing double-network hydrogel for 3D printing and strain sensor. ACS Appl. Mater. Interfaces 2017, 9, 26429-26437. [CrossRef] [PubMed]

147. Song, J.H.; Kim, Y.T.; Cho, S.; Song, W.J.; Moon, S.; Park, C.G.; Park, S.; Myoung, J.M.; Jeong, U. Surface-embedded stretchable electrodes by direct printing and their uses to fabricate ultrathin vibration sensors and circuits for 3D structures. Adv. Mater. 2017, 29, 1702625. [CrossRef] [PubMed]

148. Zhang, S.M.; Cai, L.; Li, W.; Miao, J.H.; Wang, T.Y.; Yeom, J.; Sepulveda, N.; Wang, C.A. Fully printed silver-nanoparticle-based strain gauges with record high sensitivity. Adv. Electron. Mater. 2017, 3, 1700067. [CrossRef]

149. Cai, L.; Zhang, S.M.; Zhang, Y.H.; Li, J.Q.; Miao, J.S.; Wang, Q.F.; Yu, Z.B.; Wang, C. Direct printing for additive patterning of silver nanowires for stretchable sensor and display applications. Adv. Mater. Technol. 2018, 3, 1700232. [CrossRef]

150. Wei, H.; Li, K.; Liu, W.G.; Meng, H.; Zhang, P.X.; Yan, C.Y. 3D printing of free-standing stretchable electrodes with tunable structure and stretchability. Adv. Eng. Mater. 2017, 19, 1700341. [CrossRef]

151. Muth, J.T.; Vogt, D.M.; Truby, R.L.; Menguc, Y.; Kolesky, D.B.; Wood, R.J.; Lewis, J.A. Embedded 3D printing of strain sensors within highly stretchable elastomers. Adv. Mater. 2014, 26, 6307-6312. [CrossRef] [PubMed]

152. Frutiger, A.; Muth, J.T.; Vogt, D.M.; Menguc, Y.; Campo, A.; Valentine, A.D.; Walsh, C.J.; Lewis, J.A. Capacitive soft strain sensors via multicore-shell fiber printing. Adv. Mater. 2015, 27, 2440-2446. [CrossRef] [PubMed]

153. Fuh, Y.K.; Wang, B.S.; Tsai, C.Y. Self-Powered Pressure Sensor with fully encapsulated 3D printed wavy substrate and highly-aligned piezoelectric fibers array. Sci. Rep. 2017, 7, 6759. [CrossRef] [PubMed]

154. Qin, H.T.; Cai, Y.; Dong, J.Y.; Lee, Y.S. Direct printing of capacitive touch sensors on flexible substrates by additive E-Jet printing with silver nanoinks. J. Manuf. Sci. Trans. ASME 2017, 139, 031011. [CrossRef]

(C) 2018 by the authors. Licensee MDPI, Basel, Switzerland. This article is an open access article distributed under the terms and conditions of the Creative Commons Attribution (CC BY) license (http://creativecommons.org/licenses/by/4.0/). 